"(C) 2015 IEEE. Personal use of this material is permitted. Permission from IEEE must be obtained for all other uses, in any current or future media, including reprinting/republishing this material for advertising or promotional purposes, creating new collective works, for resale or redistribution to servers or lists, or reuse of any copyrighted component of this work in other works." 


\title{
Boosting for Multi-Graph Classification
}

\author{
Jia Wu, Student Member, IEEE, Shirui Pan, Xingquan Zhu, Senior Member, IEEE, and Zhihua Cai
}

\begin{abstract}
In this paper, we formulate a novel graph-based learning problem, Multi-Graph Classification (MGC), which aims to learn a classifier from a set of labeled bags each containing a number of graphs inside the bag. A bag is labeled positive, if at least one graph in the bag is positive, and negative otherwise. Such a multi-graph representation can be used for many real-world applications, such as webpage classification, where a webpage can be regarded as a bag with texts and images inside the webpage being represented as graphs. This problem is a generalization of Multi-Instance Learning (MIL) but with vital differences, mainly because instances in MIL share a common feature space whereas no feature is available to represent graphs in a multi-graph bag. To solve the problem, we propose a boosting based multi-graph classification framework (bMGC). Given a set of labeled multi-graph bags, bMGC employs dynamic weight adjustment at both bag- and graph-levels to select one subgraph in each iteration as a weak classifier. In each iteration, the bag and graph weights are adjusted such that an incorrectly classified bag will receive a higher weight because the predicted bag label conflicts to its genuine label, whereas an incorrectly classified graph will receive a lower weight value if the graph is in a positive bag (or a higher weight if the graph is in a negative bag). Accordingly, bMGC is able to differentiate graphs in positive and negative bags to derive effective classifiers to form a boosting model for multi-graph classification. Experiments and comparisons on real-world multigraph learning tasks demonstrate the algorithm performance.
\end{abstract}

Index Terms-Graph classification, multi-instance learning, boosting, multi-graph, subgraph mining.

\section{INTRODUCTION}

Graph classification, in which the object to be classified is a graph, has found many applications in the past decade, such as chemical compounds [1], XML documents [2], program flows [3], and images [4]. Despite of its success in a broad spectrum of areas, standard graph classification setting is rather restrictive for many real-world learning problems. One of such problems is Multi-Graph Classification (MGC), in which the object to be classified is a bag of graphs. For example, a webpage may consist of texts and images, where texts can be represented as graphs to preserve contextual information [5] and images can also be represented as graphs to describe structural dependency between image regions [6]. As a result, a webpage can be regarded as a bag containing a number of graphs, each of which represents a certain part of the webpage

J. Wu is with the School of Computer Science, China University of Geosciences, Wuhan 430074, China, and the Centre for Quantum Computation and Intelligent Systems, Faculty of Engineering \& Information Technology, University of Technology Sydney, Australia (e-mail: jia.wu@student.uts.edu.au)

S. Pan is with Centre for Quantum Computation and Intelligent Systems, Faculty of Engineering \& Information Technology, University of Technology Sydney, Australia (e-mail: shirui.pan@student.uts.edu.au)

$\mathrm{X}$. Zhu is with Dept. of Computer and Electrical Engineering \& Com puter Science, Florida Atlantic University, Boca Raton, USA (e-mail: xzhu3@fau.edu)

Z. Cai is with the School of Computer Science, China University of Geosciences, Wuhan 430074, China (e-mail: zhcai@cug.edu.cn)

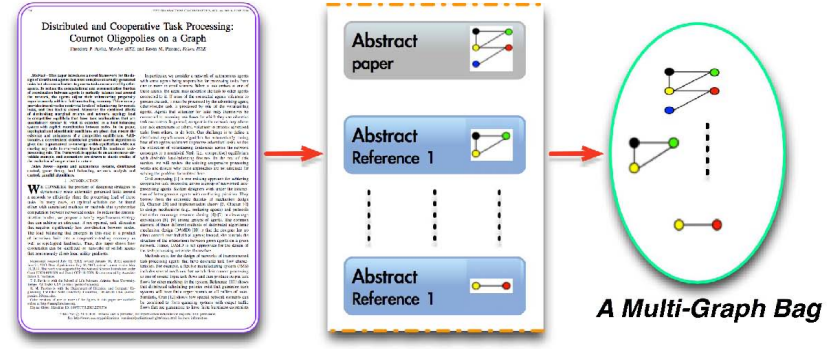

Fig. 1. An example of multi-graph representation for a scientific publication. Each paper is represented as a multi-graph bag, where each graph inside the bag corresponds to the abstract of the paper or the abstract of the reference cited in the paper (a graph is formed by using keywords of the abstract as nodes and their correlations as edges). The graph construction details are reported in Section VII-A.

content. For an information seeker, a webpage is interesting to him/her if one or multiple parts of the webpage (texts and/or images) draws his/her attention - A graph bag is positive if at least one graph in the bag is positive. On the other hand, the webpage is not interesting to the viewer if none of the content attracts the viewer - A graph bag is negative if all graphs inside the bag are negative.

The above multi-graph setting can be found useful in many other domains. For bio-pharmaceutical test, labeling individual molecules (which can be represented as graphs) is expensive and time-consuming. Molecular group activity prediction can be used to investigate the activity of a group (i.e. a bag) of molecules, with the active group (i.e. positive bag), in which at least one molecule is active, be further investigated for individual activity test. Another MGC application is scientific publication classification, where a paper and its references can be represented as a bag of graphs and each graph (i.e. a paper) is formed by using the correlations between keywords in the paper, as shown in Figure 1. A bag is labeled positive, if the paper or any of its references is relevant to a specific topic. Similarly, for online review based product recommendation, each product receives many customer reviews. For each review composed of detailed text descriptions, we can employ a graph to represent the review descriptions. Thus, a product can be represented as a bag of graphs. Assume customers mainly concern about several key properties, such as "affordability" and "durability", of the product. A product (i.e. a bag) can be labeled as positive if it receives very positive review in any of these properties, and negative otherwise. As a result, we can use MGC learning to help recommend products to customers.

Indeed, the MGC problem is a generalization of MultiInstance Learning (MIL) to graph data, but with significant complications. Existing MIL methods cannot be simply applied to the multi-graph setting because they can only handle bags with all instances being represented in a common vecto- 
rial feature space. Unfortunately, in the MGC problem setting, graphs cannot directly provide feature vectors for learning. On the other hand, existing graph classification methods cannot be used to tackle the MGC problem neither, because they require each single graph to be labeled in order to learn a classifier. One simple solution is to represent all graphs in the same feature space, by using some subgraph feature selection methods [7], [8], [9] to convert graphs as instances, and then apply existing MIL methods to the instance bags. However, this simple solution suffers from three inherent disadvantages:

- Large subgraph feature space: the graph substructure feature space increases, with respect to the number of edges and nodes, in an exponential order. It is computationally inefficient, or even infeasible, to enumerate all subgraph features, and then select some subgraph features for classification.

- Feature filtering inefficiency: by separating subgraph feature mining and feature selection into two steps, the filtering process of finding salient subgraph patterns will depend on the optimal solution of the subsequent learning algorithm. It is very difficult to theoretically guarantee that the statistical criterion provides good features for the subsequent learning algorithm. This is the problem of all filter methods (as discussed in [10]).

- Bag constraints: the bag constraints in the multi-graph learning provide important information to differentiate positive and negative graphs, whereas the simple solution directly extracts subgraphs from all graphs without considering multi-graph nature for effective learning.

In summary, the multi-graph classification problem for the aforementioned real-world applications needs to address two essential challenges: (1) Labeling Ambiguity. Labels are only available at bag level instead of instance level (i.e. a bag is labeled positive if it has at least one positive graph and negative otherwise); and (2) Structured Data Representation. Instances in a bag are not vectors but graphs, which implies that all instances are not represented in a common feature space for calculating similarities or distances.

Motivated by the above challenges, in this paper, we propose a boosting based learning framework, named bMGC, for multi-graph classification. In each boosting iteration, bMGC explores the most informative subgraph to construct a single weak classifier, which is used to update the weights of graphs and bags to obtain the next informative subgraph. At the end of the boosting process, the selected weak classifiers are combined to form a strong classifier. A unique characteristic of bMGC is that it combines bag- and graph-level constraints to assess the informativeness score of a subgraph. By adapting the score as a pruning criterion, we combine subgraph mining and informative subgraph exploration to dynamically construct weak classifiers on the fly. As a result, the proposed learning framework not only addresses the "labeling ambiguity" issue by using a novel two-level (bag and graph) weighting strategy but also addresses the "structured data representation" issue through a dynamic subgraph selection criterion. The experimental results on real-world data demonstrate that bMGC is effective for multi-graph classification.
The remainder of the paper is organized as follows. A brief review of related works is reported in Section II. The problem definition and the overall framework are described in Section III and IV, respectively. Section V introduces the proposed subgraph selection criterion. The bMGC algorithm is presented in Section VI, followed by the experiments in Section VII. Section VIII discusses the properties of the proposed bMGC, and we conclude the paper in Section IX.

\section{RELATED WORK}

\section{A. Multi-Instance Learning}

Multi-graph classification is a generalization of the multiinstance learning (MIL) problem, which was first proposed by Dietterich et al. [11] for drug activity prediction. Since then, it has drawn increasing interest in the machine learning community for many real-world applications, such as image categorization [12], web mining [13], language recognition [14], and computer security [15]. The key assumption of MIL formulation is that the training set is composed of some labeled bags, each of which contains a number of instances. A bag is labeled positive if at least one of its instances is positive and negative otherwise. The goal of MIL is to predict the label of an unknown bag. To aim this, several off-the-shelf methods have been developed to solve the MIL problem, which can roughly be divided into two categories.

1) Single-Instance Learner Based MIL: One approach to solve MIL problems is to upgrade generic single-instance learning methods to deal with multi-instance data. For example, lazy learning Citation-KNN and Bayesian-KNN [16] extend the $k$-nearest neighbor (KNN) algorithm for MIL. Tree learning MITI [17] and MIRI [18] are variations of decision trees for MIL. Rule learning RIPPER-MI adapts the RIPPER algorithm [19] for MIL. Neural network BP-MIP extends standard neural networks [20], and kernel method MISMO adapts the classical support vector machine [21] for MIL. Logistic learning MILR [22] applies the logistic regression to MIL, and ensemble approaches [23], [24] which extend bagging and boosting [25] to multi-instance learning.

2) Bag-Based MIL Algorithms: The first specifically designed method for MIL is the axis-parallel rectangle (APR) algorithm [11], which approximates the axis-parallel rectangles constructed by the conjunction of features. Based on the idea of APR, a number of algorithms have also been designed for MIL. Examples include Diverse Density (DD) [26], which searches a point in the feature space by maximizing the diverse density function that measures a co-occurrence of similar instances from different positive bags; MIEMDD [27], which combines the conception of Expectation-Maximization (EM) algorithm with DD to search the most likely concept; and MIOptimalBall [28], another boosting optimal ball based approach, which uses balls (with respect to various metrics) as weak hypotheses centered at instances of positive bags.

\section{B. Graph Classification}

The MGC problem can also be viewed as a generalization of graph classification where objects are bags of graphs (instead of individual graphs). Existing graph classification methods can be broadly classified into the following two categories: 
1) Global distance based approaches: The global distance based methods consider correlations [29] or similarities between two graphs and plug the kernel matrix into a off-theshelf learner, such as support vector machines, to learn a model for graph classification. Examples include e.g., graph kernels [30], [31], graph embedding [32], and graph transformation [33]. One obvious drawback of global distance based approaches is that the distance is calculated based on the similarity of global graph structures, such as random walks or paths, between two graphs. Therefore, it is not clear which substructures (or which parts of the graph) are mostly discriminative for differentiating graphs between different classes.

2) Local subgraph feature based approaches: For many graph classification tasks, such as chemical compound classification [1], research has shown that graphs within the same class may not have high global similarity but merely share some unique substructures. Accordingly, extracting important subgraph features, using some predefined criteria, to represent a graph in a vectorial space becomes a popular solution for graph classification. The most common subgraph selection criterion is frequency, which intends to select frequently appearing subgraphs by using frequent subgraph mining methods. For example, one of the most popular algorithms for frequent subgraph mining is gSpan [34]. Other methods include AGM [35], FSG [7], $\mathrm{MoFa}$ [36], and Gaston [37]. The subgraph feature mining approach seems applicable to the MGC problem as a preprocessing step to transform all graphs into feature vectors. However, one major deficiency of this approach is that it is computationally demanding to enumerate enormous frequent subgraphs in the target graph set, which inhibits its ability to handle large graph sets.

To overcome this drawback, some supervised subgraph feature extraction approaches have been developed, such as LEAP [38], gPLS [39] and COPK [8], which search directly for discriminative subgraph patterns for classification. Moreover, Jin et al. [40] proposes an efficient graph classification method using evolutionary computation for mining discriminative subgraphs for graph classification in large databases. Besides, some graph boosting methods [41], [42], [43], [44] also exist to use each single subgraph feature as a weak classifier to build boosting algorithm, including some other types of boosting approaches [45], [46] for graph classification.

\section{Problem Definition}

In this section, we define important notations and concepts, which will be used throughout the paper. We also formally define the MGC problem in this section.

Definition 1. (Connected Graph) A graph is represented as $G=(\mathcal{V}, E, \mathcal{L}, l)$ where $\mathcal{V}$ is a set of vertices, $E \subseteq \mathcal{V} \times \mathcal{V}$ is a set of edges, and $\mathcal{L}$ is the set of symbols for the vertices and edges. $l: \mathcal{V} \cup E \rightarrow \mathcal{L}$ is the function assigning labels to the vertices and edges. A connected graph is a graph such that there is a path between any pair of vertices.

Definition 2. (Bag of Graphs) A graph bag contains a number of graphs, denoted by $B_{i}=\left\{G_{1}^{i}, \ldots, G_{n_{i}}^{i}\right\}$, where $G_{j}^{i}$ and $n_{i}$ denote the jth graph and the total number of graphs in the

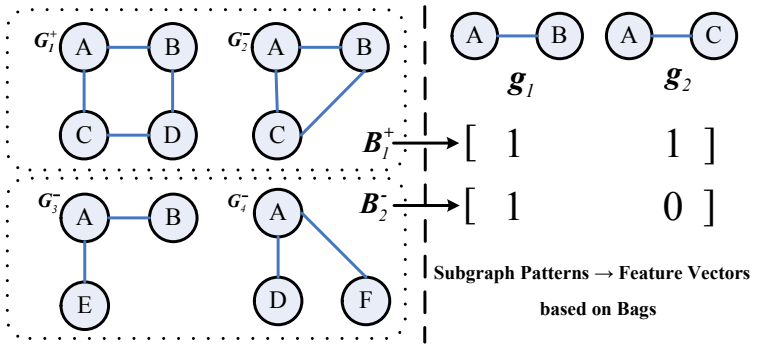

Fig. 2. An example of subgraph feature representation for bags. $B_{1}^{+}$and $B_{2}^{-}$ are positive and negative bags, respectively. $G_{1}^{+}$is a positive graph and $G_{2}^{-}$, $G_{3}^{-}$, and $G_{4}^{-}$are labeled negative. The feature value of a bag corresponding to each subgraph $g_{1}$ or $g_{2}$ is set to 1 , iff there is a graph in the bag contains the subgraph, and 0 otherwise.

ith bag, respectively. For ease of representation, we also use $G_{j}$ to denote the $j$ th graph in a given bag. A bag $B_{i}$ 's label is denoted by $y_{i} \in\{-1,+1\}$. A bag is either positive $\left(B_{i}^{+}\right)$ or negative $\left(B_{i}^{-}\right)$.

In this paper, we use $\mathcal{B}=\left\{B_{1}, \ldots, B_{p}\right\}$ to denote a set of bags associated with the weights $\mathbf{w}^{B}=\left\{w_{1}^{B}, \ldots, w_{p}^{B}\right\}$, where $p$ denotes the number of all bags in $\mathcal{B}$. We can also aggregate all graphs in $\mathcal{B}$ as $\mathcal{G}=\left\{G_{1}, \ldots, G_{q}\right\}$ associated with the weights $\mathbf{w}^{G}=\left\{w_{1}^{G}, \ldots, w_{q}^{G}\right\}$, where $q$ denotes the number of graphs in $\mathcal{G}$. Similarly, the set of positive bags in $\mathcal{B}$ is denoted by $\mathcal{B}^{+}$, with $\mathcal{B}^{-}$denoting the set of negative bags.

Definition 3. (Subgraph) Let $G=(\mathcal{V}, E, \mathcal{L}, l)$ and $g_{k}=$ $\left(\mathcal{V}^{\prime}, E^{\prime}, \mathcal{L}^{\prime}, l^{\prime}\right)$ each denotes a connected graph. $g_{k}$ is a subgraph of $G$, i.e., $g_{k} \subseteq G$, iff there exists an injective function $\varphi: \mathcal{V}^{\prime} \rightarrow \mathcal{V}$ s.t. (1) $\forall v \in \mathcal{V}^{\prime}, l^{\prime}(v)=l(\varphi(v))$; (2) $\forall(u, v) \in E^{\prime},(\varphi(u), \varphi(v)) \in E$ and $l^{\prime}(u, v)=l(\varphi(u), \varphi(v))$. If $g_{k}$ is a subgraph of $G$, then $G$ is a supergraph of $g_{k}$.

Definition 4. (Subgraph Feature Representation for Graph) Let $\mathcal{S}_{g}=\left\{g_{1}, \ldots, g_{s}\right\}$ denote a set of subgraph patterns discovered from a given set of graphs. For each graph $G_{i}$, we use a subgraph feature vector $\mathbf{x}_{i}^{G}=\left[\left(x_{i}^{g_{1}}\right)^{G}, \ldots,\left(x_{i}^{g_{s}}\right)^{G}\right]^{\top} \in$ $\{0,1\}^{s}$ to represent $G_{i}$ in the feature space, where $\left(x_{i}^{g_{k}}\right)^{G}=$ 1, iff $g_{k}$ is a subgraph of $G_{i}$ (i.e. $g_{k} \subseteq G_{i}, g_{k} \in S_{g}$ ) and $\left(x_{i}^{g_{k}}\right)^{G}=0$ otherwise.

Definition 5. (Subgraph Feature Representation for Bag) Given a set of subgraphs $\mathcal{S}_{g}=\left\{g_{1}, \cdots, g_{s}\right\}$, a graph bag $B_{i}$ can be represented by a feature vector $\mathbf{x}_{i}^{B}=$ $\left[\left(x_{i}^{g_{1}}\right)^{B}, \ldots,\left(x_{i}^{g_{s}}\right)^{B}\right]^{\top} \in\{0,1\}^{s}$, where $\left(x_{i}^{g_{k}}\right)^{B}=1$, iff $g_{k}$ is a subgraph of any graph $G_{j}$ in bag $B_{i}$ (i.e. $\exists G_{j} \in B_{i} \wedge G_{j} \supseteq$ $\left.g_{k}, g_{k} \in S_{g}\right)$ and $\left(x_{i}^{g_{k}}\right)^{B}=0$ otherwise.

An example of subgraph feature representation for graph bags is illustrated in Figure 2, where two graph bags ( $B_{1}^{+}$and $B_{2}^{-}$on the left panel) are represented as two 2-dimensional feature vectors (on the right panel) based on two subgraph patterns $\left(g_{1}\right.$ and $\left.g_{2}\right)$.

Given a multi-graph set $\mathcal{B}$ with a number of labeled graph bags, where each positive bag contains at least one positive graph and all graphs in each negative bag are negative (i.e. the bag constraint in MGC), the aim of multi-graph classification is to build a prediction model from the training multi-graph 
bag set $\mathcal{B}$ to predict some previously unseen graph bags with unknown label with maximum bag classification accuracy.

\section{OVERALl Framework of bMGC}

In multi-graph bags, there is no feature available to represent graphs, so existing multi-instance learning (MIL) methods, which require instances to have a vectorized feature representation, cannot be applied to MGC. In addition, due to lack of labeling information for individual graphs inside positive bags, subgraph feature based graph classification cannot be directly applied to MGC neither.

To solve the above issues, in this section we propose a boosting based multi-graph classification framework (bMGC). The framework applies dynamic weight adjustment at both graph- and bag-levels to select one subgraph in each iteration to construct a single weak classifier. In each iteration, the bag and graph weights are adjusted by the last bag-level and graphlevel weak classifiers, respectively. By doing so, bMGC is able to differentiate graphs in positive or negative bags to derive effective learning models by boosting all the single subgraph bag-level weak classifiers. The proposed bMGC framework, as shown in Figure 3, includes the following four major steps:

- Subgraph Candidate Generation: Generating subgraph candidates is a key step for selecting the most informative subgraph. To find subgraph candidates with diverse structures, we aggregate graphs in multi-graph bags into three graph sets: (1) graphs in all bags, (2) graphs in all positive bags, and (3) graphs in all negative bags. A gSpan [34] based subgraph mining procedure is performed on each graph set, through which a set of diverse subgraph candidate patterns can be discovered for validation.

- Bag Constrained Subgraph Exploration: In the $t$ th iteration, an informative subgraph $g_{t}$ is selected to form a weak classifier for MGC under the weighted bag- and graph-level constraints. To obtain the $t+1$ th informative subgraph, the weights of bags and graphs should be updated. After $m$ iterations, the selected $m$ subgraphs will correspond to $m$ weak classifiers for learning.

- Updating Weights of Bags and Graphs: After we find the $t$ th informative subgraph $g_{t}$, a bag-level classifier $\mathcal{H}_{t}^{B}$ and a graph-level classifier $\mathcal{H}_{t}^{G}$ will be trained respectively. For graphs, due to our assumption that we apply bag labels to graphs, some graphs in positive bags have been assigned wrong labels. If a graph $G_{i}$ in positive bag set $\mathcal{B}^{+}$is misclassified by $\mathcal{H}_{t}^{G}$, in the next iteration we will decrease $G_{i}$ 's weight to reduce its impact in the learning process. If a graph $G_{i}$ in negative bag set $\mathcal{B}^{-}$is misclassified, its weight will be increased, such that $G_{i}$ in the negative bag set will play a more important role to help the learning algorithm find better subgraphs.

- Boosting Classification: After the subgraphs are selected in all iterations to form the corresponding single weak classifiers, they can be weighted to construct a strong classifier for multi-graph classification.

In the following two sections, we first propose our subgraph exploration criterion in Section $\mathrm{V}$ and then introduce detailed procedures of bMGC in Section VI.

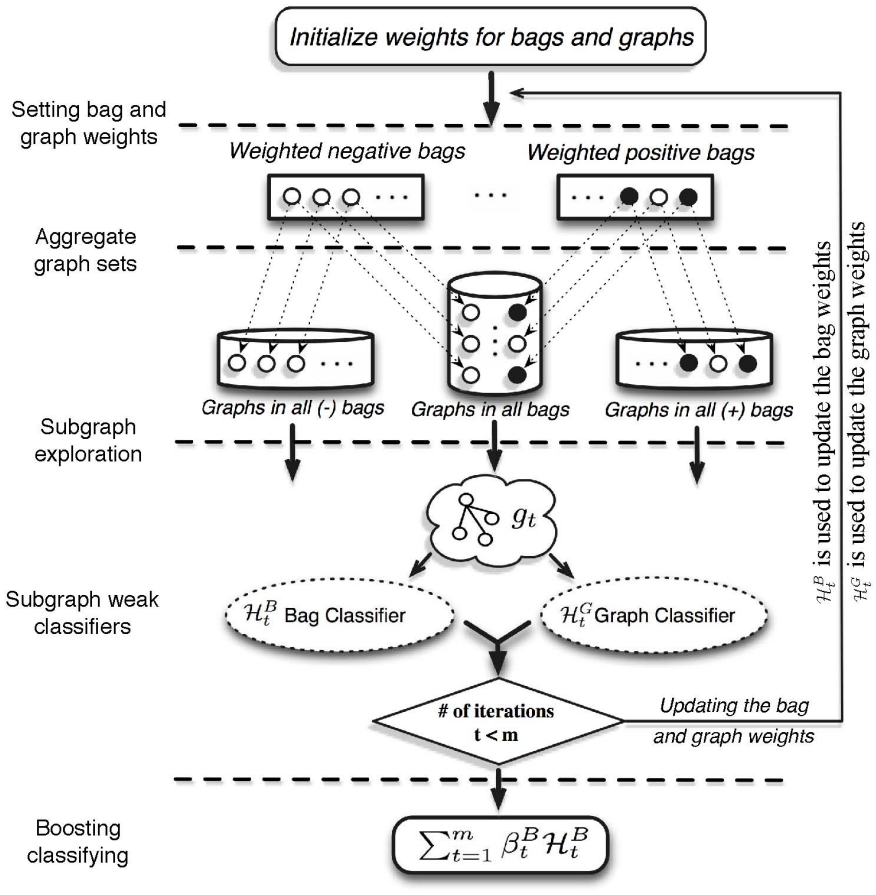

Fig. 3. An overview of the proposed bMGC framework.

\section{SUbGRAPH EXPLORATION}

Exploring optimal bag constrained subgraph in each iteration of bMGC is a non-trivial task. This process has two main challenges: (1) How to utilize the information of the labeled graphs in negative bags? (2) How to tackle the problem that the labels of graphs in positive bags are unknown? Assume that a set of candidate graphs are collected from the bag set $\mathcal{B}$, let $\mathcal{S}_{g}$ denote the complete set of subgraphs in $\mathcal{B}$, and $g_{t}$ be the optimal subgraph selected from $\mathcal{S}_{g}$ in the $t$ th iteration. Our bag constrained subgraph exploration aims to find the most informative subgraph $g_{t}$ in each iteration with the updating weights for both bags and graphs. Let $\mathcal{Z}\left(g_{k}\right)$, the evaluation criterion for a single subgraph $g_{k} \in \mathcal{S}_{g}$, be a function to measure the informativeness of $g_{k}$ as

$$
g_{t}=\arg \max _{g_{k} \in \mathcal{S}_{g}}\left(\mathcal{Z}\left(g_{k}\right)\right)
$$

The objective function in Eq. (1) indicates that the optimal bag constrained subgraph $g_{t}$ should have the maximum discriminative capability for multi-graph classification.

\section{A. Evaluation Criterion for Subgraphs}

In order to measure the informativeness of a subgraph $g_{k}$, i.e., $\mathcal{Z}\left(g_{k}\right)$, such that we can discover the most informative subgraph for bags, we impose constraints to the labeled bags in the multi-graph bag set $\mathcal{B}$, through which the subgraph selection criterion $\mathcal{Z}\left(g_{k}\right)$ can be properly defined. For two bags, $B_{i}$ and $B_{j}$, if they have the same class labels, there is a pairwise must-link constraint between them. If $B_{i}$ and $B_{j}$ have different class labels, there is a cannot-link constraint between them. To further take the data distributions in each bag into consideration, we also add graph-level constraints to ensure that the selected subgraphs can make graphs in each negative 
bag close to each other and make graphs in each positive bag be maximally separated. In summary, a good subgraph should satisfy the following constraints.

- Weighted Bag Must-Link: If there is a must-link between $B_{i}$ and $B_{j}$, their subgraph feature vectors $\mathbf{x}_{i}^{B}$ and $\mathbf{x}_{j}^{B}$ should be close to each other. In a MGC scenario, each bag $B_{i}$ is associated with a weight $w_{i}^{B}$. For each pair of bags with the same class label, the selected subgraph should ensure that bags with similar weights (analogous to importance) have a high similarity.

- Weighted Bag Cannot-Link: If there is a cannot-link between $B_{i}$ and $B_{j}$, the underlying subgraph feature vectors $\mathbf{x}_{i}^{B}$ and $\mathbf{x}_{j}^{B}$ should be distinct from each other. For each pair of bags in different classes, the smaller the weight difference between the two bags, the more impact the constraint will have for selecting subgraph to represent the distinctness between them.

- Weighted Graph Must-Link: If there is a must-link between $G_{i}$ and $G_{j}$, their subgraph feature vectors $\mathbf{x}_{i}^{G}$ and $\mathrm{x}_{j}^{G}$ should be close to each other. In bMGC, only graphs in negative bags are known to have genuine labels, in which the feature representations of the two weighted graphs should have low diversity.

- Weighted Graph Separability: If genuine labels of graphs $G_{i}$ and $G_{j}$ are unknown, the corresponding subgraph feature vectors $\mathbf{x}_{i}^{G}$ and $\mathbf{x}_{j}^{G}$ should be different. It is similar to the Principal Component Analysis (PCA)'s assumption [47], which aims to find the component with largest possible variance. For graphs in positive bags, at least one is positive, but actually we do not know which one or more are positive. Thus, a reasonable solution is that we make the weighted graphs in a positive bag have more diversity. Similar assumption has also been used in [9] to handle unlabeled graphs in a semi-supervised learning setting.

In summary, the bag must-link and bag cannot-link constraints are applied to bags with the same label and different labels, respectively. While the graph must-link and graph separability constraints are only applied to graphs in negative bags and graphs in positive bags, respectively.

By imposing constraints at both bag and graph levels, our evaluation criterion can effectively capture informative subgraphs underlying the multi-graph classification. Based on the above considerations, we derive a criterion $\mathcal{Z}\left(g_{k}\right)$ for measuring the informativeness of a subgraph $g_{k}$ as follows:

$$
\begin{aligned}
\mathcal{Z}\left(g_{k}\right) & =\frac{1}{2 A} \sum_{y_{\mathrm{i}} y_{j}=-1}\left(\mathcal{D}_{g_{k}} w_{i}^{B} \mathbf{x}_{i}^{B}-\mathcal{D}_{g_{k}} w_{j}^{B} \mathbf{x}_{j}^{B}\right)^{2} \\
& -\frac{1}{2 B} \sum_{y_{\mathrm{i}} y_{j}=1}\left(\mathcal{D}_{g_{k}} w_{i}^{B} \mathbf{x}_{i}^{B}-\mathcal{D}_{g_{k}} w_{j}^{B} \mathbf{x}_{j}^{B}\right)^{2} \\
& -\frac{1}{2 C} \sum_{\forall G_{i}, G_{j} \in \mathcal{B}^{-}}\left(\mathcal{D}_{g_{k}} w_{i}^{G} \mathbf{x}_{i}^{G}-\mathcal{D}_{g_{k}} w_{j}^{G} \mathbf{x}_{j}^{G}\right)^{2} \\
& +\frac{1}{2 D} \sum_{\forall G_{i}, G_{j} \in \mathcal{B}^{+}}\left(\mathcal{D}_{g_{k}} w_{i}^{G} \mathbf{x}_{i}^{G}-\mathcal{D}_{g_{k}} w_{j}^{G} \mathbf{x}_{j}^{G}\right)^{2}
\end{aligned}
$$

where $w_{i}^{B}, w_{j}^{B}, w_{i}^{G}$, and $w_{j}^{G}$ are the weights for $B_{i}, B_{j}$, $G_{i}$, and $G_{j}$, respectively. $\mathcal{D}_{g_{k}}=\operatorname{diag}\left(d\left(g_{k}\right)\right)$ is a diago- nal matrix indicating which subgraph feature $g_{k}$ is selected from $S_{g}$ to represent the bags or graphs, $d\left(g_{k}\right)_{i}=I\left(g_{i}=\right.$ $g_{k}, g_{i} \in S_{g}$ ) with $I(\cdot)$ equaling to 1 if the condition inside is true and 0 otherwise. $A=\sum_{y_{i} y_{j}=-1} 1, B=\sum_{y_{i} y_{j}=1} 1$, $C=\sum_{G_{i}, G_{j} \in \mathcal{B}^{-}} 1$, and $D=\sum_{G_{i}, G_{j} \in \mathcal{B}^{+}} 1$ assess the total pairwise sets of constraints in the bag cannot-link, bag mustlink, graph must-link and graph separability.

We define two matrices for bag-level and graph-level constraints, denoted by $M_{B}=\left[M_{i j}^{B}\right]^{p \times p}$ and $M_{G}=\left[M_{i j}^{G}\right]^{q \times q}$, respectively, where $M_{i j}^{B}=\left\{1 / A, y_{i} y_{j}=-1 ;-1 / B, y_{i} y_{j}=1\right\}$, and $M_{i j}^{G}=\left\{-1 / C, \forall G_{i}, G_{j} \in \mathcal{B}^{-} ; 1 / D, \forall G_{i}, G_{j} \in \mathcal{B}^{+}\right\}$.

As a result, Eq. (2) can be rewritten as

$$
\begin{aligned}
\mathcal{Z}\left(g_{k}\right) & =\mathcal{Z}\left(g_{k}\right)^{B}+\mathcal{Z}\left(g_{k}\right)^{G} \\
& =\frac{1}{2} \sum_{y_{i} y_{j}}\left(\mathcal{D}_{g_{k}} w_{i}^{B} \mathbf{x}_{i}^{B}-\mathcal{D}_{g_{k}} w_{j}^{B} \mathbf{x}_{j}^{B}\right)^{2} M_{i j}^{B} \\
& +\frac{1}{2} \sum_{G_{i}, G_{j}}\left(\mathcal{D}_{g_{k}} w_{i}^{G} \mathbf{x}_{i}^{G}-\mathcal{D}_{g_{k}} w_{j}^{G} \mathbf{x}_{j}^{G}\right)^{2} M_{i j}^{G}
\end{aligned}
$$

For bag-level evaluation $\mathcal{Z}\left(g_{k}\right)^{B}$, we have

$$
\begin{aligned}
\mathcal{Z}\left(g_{k}\right)^{B} & =\frac{1}{2} \sum_{y_{i} y_{j}}\left(\mathcal{D}_{g_{k}} w_{i}^{B} \mathbf{x}_{i}^{B}-\mathcal{D}_{g_{k}} w_{j}^{B} \mathbf{x}_{j}^{B}\right)^{2} M_{i j}^{B} \\
& =\operatorname{tr}\left(\mathcal{D}_{g_{k}}^{\top} X_{B} W_{B}\left(D_{B}-M_{B}\right) W_{B}^{\top} X_{B}^{\top} \mathcal{D}_{g_{k}}\right) \\
& =\operatorname{tr}\left(\mathcal{D}_{g_{k}}^{\top} X_{B} W_{B} L_{B} W_{B}^{\top} X_{B}^{\top} \mathcal{D}_{g_{k}}\right) \\
& =\left(\boldsymbol{f}_{g_{k}}^{B}\right)^{\top} W_{B} L_{B} W_{B}^{\top} \boldsymbol{f}_{g_{k}}^{B} \\
& =\left(\boldsymbol{f}_{g_{k}}^{B}\right)^{\top} Q_{B} \boldsymbol{f}_{g_{k}}^{B}
\end{aligned}
$$

where $L_{B}=D_{B}-M_{B}$ is a Laplacian matrix, where $D_{B}=\operatorname{diag}\left(d_{i}^{B}\right)$ is a diagonal matrix with $d_{i}^{B}=\sum_{j} M_{i j}^{B}$. $Q_{B}=W_{B} L_{B} W_{B}^{\top}$, where $W_{B}$ is a also a diagonal matrix, with $W_{i i}^{B}=w_{i}^{B}$ denoting the weight of the $i$ th bag $B_{i}$. $X_{B}=\left[\mathbf{x}_{1}^{B}, \ldots, \mathbf{x}_{p}^{B}\right]=\left[\boldsymbol{f}_{g_{1}}^{B}, \cdots, \boldsymbol{f}_{g_{s}}^{B}\right]^{\top} \in\{0,1\}^{s \times p}$, where $\boldsymbol{f}_{g_{k}}^{B}$ is an indicator vector of subgraph $g_{k}$ with respect to all the bags in $\mathcal{B}$. Specifically, $\boldsymbol{f}_{g_{k}}^{B}=\left[f_{g_{k}}^{B_{1}}, \ldots, f_{g_{k}}^{B_{p}}\right]^{\top} \in\{0,1\}^{p}$, where $f_{g_{k}}^{B_{i}}=1$ iff $\exists G \in B_{i} \wedge G \supseteq g_{k}$ and $f_{g_{k}}^{B_{i}}=0$ otherwise.

Similarly, the graph-level evaluation $\mathcal{Z}\left(g_{k}\right)^{G}$ can be rewritten in the form of matrix. Taking both the bag-level and graphlevel evaluation functions together, we have

$$
\begin{aligned}
\mathcal{Z}\left(g_{k}\right) & =\mathcal{Z}\left(g_{k}\right)^{B}+\mathcal{Z}\left(g_{k}\right)^{G} \\
& =\left(\boldsymbol{f}_{g_{k}}^{B}\right)^{\top} Q_{B} \boldsymbol{f}_{g_{k}}^{B}+\left(\boldsymbol{f}_{g_{k}}^{G}\right)^{\top} Q_{G} \boldsymbol{f}_{g_{k}}^{G} \\
& =\boldsymbol{f}_{g_{k}}^{\top} Q \boldsymbol{f}_{g_{k}}
\end{aligned}
$$

where $Q_{G}=W_{G} L_{G} W_{G}^{\top}$, with $W_{G}$ a diagonal matrix, i.e., $W_{i i}^{G}=w_{i}^{G}$, denoting the weight of the $i$ th graph $G_{i}$. $L_{G}=D_{G}-M_{G}$ is known as a Laplacian matrix, where $D_{G}=\operatorname{diag}\left(d_{i}^{G}\right)$ is a diagonal matrix with $d_{i}^{G}=\sum_{j} M_{i j}^{G}$. Meanwhile, $\boldsymbol{f}_{g_{k}}^{G}$ is an indicator vector, of which subgraph $g_{k}$ with respect to all graphs in $\mathcal{G}$, and $\boldsymbol{f}_{g_{k}}^{G}=\left[f_{g_{k}}^{G_{1}}, \cdots, f_{g_{k}}^{G_{q}}\right]^{\top} \in$ $\{0,1\}^{q}$, where $f_{g_{k}}^{G_{i}}=1$ iff $g_{k} \subseteq G_{i}$ and $f_{g_{k}}^{G_{i}}=0$ otherwise.

According to Eq. (5), it is

$$
\boldsymbol{f}_{g_{k}}=\left[\begin{array}{l}
\boldsymbol{f}_{g_{k}}^{B} \\
\boldsymbol{f}_{g_{k}}^{G}
\end{array}\right], \quad Q=\left[\begin{array}{cc}
Q_{B} & 0 \\
0 & Q_{G}
\end{array}\right]
$$

where $\boldsymbol{f}_{g_{k}}$ is an indicator vector of subgraph $g_{k}$ with respect to the data combined with bag matrix $X_{B}$ and graph matrix 
$X_{G}$. By denoting the function as $h\left(g_{k}, Q\right)=\boldsymbol{f}_{g_{k}}^{\top} Q \boldsymbol{f}_{g_{k}}$, the problem of maximizing $\mathcal{Z}\left(g_{k}\right)$ in Eq. (1) is equivalent to finding a subgraph that can maximize the $h\left(g_{k}, Q\right)$, which can be represented as:

$$
g_{t}=\max _{g_{k} \in \mathcal{S}_{g}} h\left(g_{k}, Q\right)
$$

Definition 6. (bScore) Given two matrices $M_{B}$ and $M_{G}$ embedding the label information, respectively, and two corresponding weight matrices $W_{B}$ and $W_{G}$, the informativeness score of a subgraph $g_{k}$ is defined in Eq. (8).

$$
r\left(g_{k}\right)=h\left(g_{k}, Q\right)=\boldsymbol{f}_{g_{k}}^{\top} Q \boldsymbol{f}_{g_{k}}
$$

In the above definition, a larger bScore $r\left(g_{k}\right)$ value represents a stronger dependency between this subgraph feature and the corresponding labels. In other words, good subgraph features should have high bScore values. To find the optimal subgraph in each iteration, we can calculate bScore values of all subgraphs in $\mathcal{S}_{g}$, and then select the topmost subgraph with the highest $r\left(g_{k}\right)$ value.

\section{B. Upper Bound of bScore}

Before we introduce detailed algorithm to mine the optimal subgraph in each iteration, we derive a bScore upper bound to help prune the subgraph search space. A convenient method to compute a upper bound of bScore value is given as follows:

Theorem 1. Given two subgraphs $g_{k}, g_{k}{ }^{\prime} \in S_{g}, g_{k}^{\prime}$ is a supergraph of $g_{k}$ (i.e. $\left.g_{k}^{\prime} \supseteq g_{k}\right)$. The bScore value $g_{k}^{\prime}\left(r\left(g_{k}^{\prime}\right)\right)$ is bounded by $\hat{r}\left(g_{k}\right)$, i.e., $r\left(g_{k}^{\prime}\right) \leq \hat{r}\left(g_{k}\right)$ :

$$
\hat{r}\left(g_{k}\right)=\boldsymbol{f}_{g_{k}}^{\top} \hat{Q} \boldsymbol{f}_{g_{k}}
$$

where $\hat{Q}=\left[\begin{array}{cc}\hat{Q}_{B} & 0 \\ 0 & \hat{Q}_{G}\end{array}\right]$, in which $\hat{Q}_{B}$ and $\hat{Q}_{G}$ are defined as $\hat{Q}_{i j}^{B}=\max \left(0, Q_{i j}^{B}\right)$ and $\hat{Q}_{i j}^{G}=\max \left(0, Q_{i j}^{G}\right)$.

For any $g_{k}^{\prime} \supseteq g_{k}, r\left(g_{k}^{\prime}\right) \leq \hat{r}\left(g_{k}\right)$. The corresponding proof is given in Appendix A.

\section{Mining Bag Constrained Subgraph}

For subgraph selection, we employ a depth-first search (DFS) based algorithm gSpan [34] to enumerate subgraphs. The key idea of gSpan is that each subgraph has a unique DFS code, which is defined by a lexicographic order of the discovery time during the search process. Two subgraphs are isomorphism iff they have the same minimum DFS code. By employing a depth-first search strategy on the DFS code tree (where each node is a subgraph), gSpan can enumerate all frequent subgraphs efficiently.

Algorithm 1 reports the proposed bag constrained subgraph exploration process, which starts with an empty optimal subgraph set and continuously enumerates subgraphs by recursively visiting the DFS code tree. If a subgraph $g_{k}$ is not a frequent subgraph, both $g_{k}$ and its subtree will be pruned (lines 3-5), in which freq $\left(g_{k}\right)$ denotes the percentage of graphs containing the subgraph $g_{k}$ in graph dataset $\mathcal{G}$; otherwise, we calculate $g_{k}$ 's bScore value $r\left(g_{k}\right)$ (line 6). If $r\left(g_{k}\right)$ is larger than the

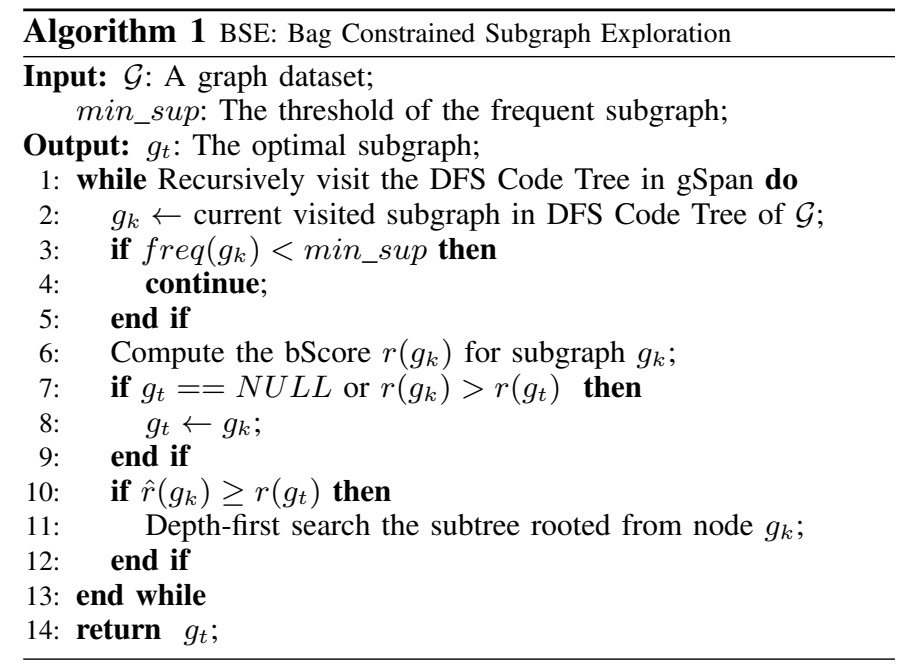

current optimal score $r\left(g_{t}\right)$ or it is the first step (i.e. the optimal subgraph set is empty), we regard $g_{k}$ as the current optimal subgraph $g_{t}$ (lines 7-9). After that, the upper bound pruning module will check if $\hat{r}\left(g_{k}\right)$ is less than $r\left(g_{t}\right)$, if so, it means that the bScore value of any supergraph $g_{k}^{\prime}$ of $g_{k}$ (i.e. $g_{k}^{\prime} \supseteq g_{k}$ ) will not be greater than $r\left(g_{t}\right)$. Thus, we can safely prune subtrees rooted from $g_{k}$ in the search space. If $\hat{r}\left(g_{k}\right)$ is indeed greater than the bScore of $g_{t}$, we cannot prune this space since there might exist a supergraph $g_{k}^{\prime} \supseteq g_{k}$ that $r\left(g_{k}^{\prime}\right) \geq r\left(g_{t}\right)$, so the depth-first search will continue by following the children of $g_{k}$ (lines 10-12), until the frequent subgraph mining process is completed.

\section{VI. bMGC}

The detailed procedures of bMGC are reported in Algorithm 2, which iteratively expands the candidate graph set to exact informative subgraphs, then explores the optimal subgraphs based on bScore. After $m$ iterations, bMGC boosts the $m$ selected weak classifiers to obtain the final classification model.

bMGC Algorithm: In Algorithm 2, bMGC differentiates and considers graph in three sets: graphs in positive bags $\mathcal{G}^{+}$, graphs in negative bags $\mathcal{G}^{-}$, and graphs in both positive and negative bags $\mathcal{G}$. The benefit of this process that considering positive and negative bags separately will increase the candidate graph set for exploring subgraphs. By doing so, the subgraph space becomes more dense, through which a good subgraph can be discovered.

The "while" loop in Algorithm 2 represents the boosting process of bMGC. In each iteration, the subgraph mining is carried out on three graph sets as shown from lines 7 to 9 . The current optimal subgraph $g_{t}$ is the one with the highest bScore with respect to the subgraph discovered from each individual graph sets (line 10). In bMGC, the subgraph $g_{t}$ is directly used as a weak bag classifier $\mathcal{H}_{t}^{B}$ or a weak graph classifier $\mathcal{H}_{t}^{G}$, where $\mathcal{H}_{t}^{B}\left(B_{i}\right)=1$ iff $\left(x_{i}^{g_{t}}\right)^{B}=1$, and $\mathcal{H}_{t}^{B}\left(B_{i}\right)=-1$, otherwise. The same classification method is used in graph based subgraph classifier $\mathcal{H}_{t}^{G}$. Accordingly, the steps from lines 11 to 20 use the error rates of the weak classifiers to updated the parameters of the boosting framework. 


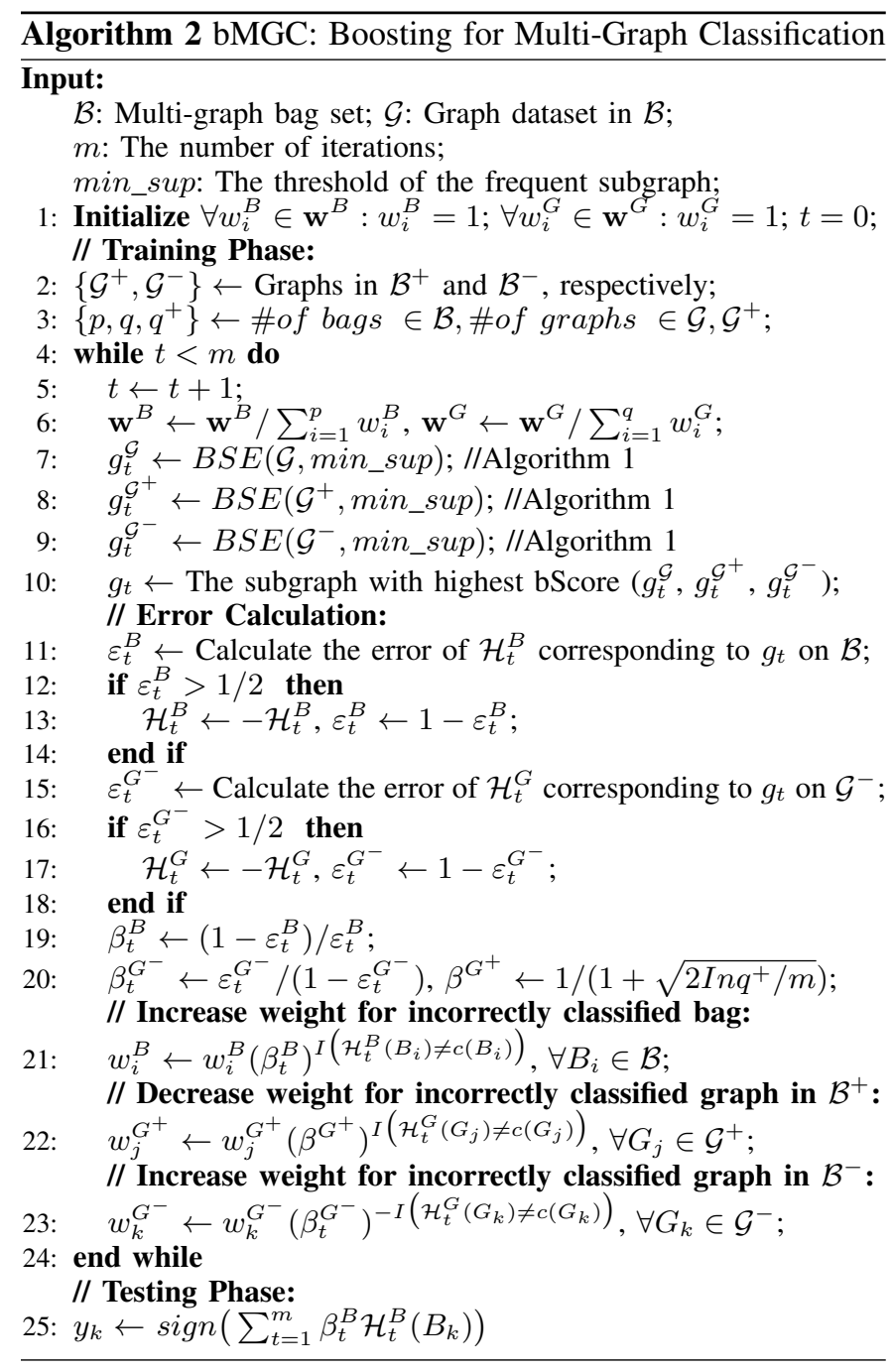

Updating bag and graph weights: To obtain the $t+1$ th optimal subgraph $g_{t+1}$, we must update the weights of bags and graphs using the $t$ th optimal subgraph $g_{t}$. The error $\varepsilon_{t}^{B}$ (line 11 ) on a bag set $\mathcal{B}$ can be defined as follows:

$$
\varepsilon_{t}^{B}=\sum_{i=0}^{p} \frac{w_{i}^{B} I\left(\mathcal{H}_{t}^{B}\left(B_{i}\right) \neq c\left(B_{i}\right)\right)}{\sum_{i=1}^{p} w_{i}^{B}}
$$

where $c\left(B_{i}\right)$ returns the label for the $i$ th bag and $I(\cdot)$ is the indicator function. Similarly, The error $\varepsilon_{t}^{G^{-}}$(line 15) on a negative graph set can be obtained. Note that $\varepsilon_{t}^{B}$ and $\varepsilon_{t}^{G^{-}}$ are required to be smaller than $1 / 2$. If not, the underlying classifier is worse than random hypothesis, and then we should use $-\mathcal{H}_{t}^{B}$ and $-\mathcal{H}_{t}^{G}$ to replace the current bag- and graphlevel classifiers, respectively. As a result, the underlying errors on bag set and negative graph set become $1-\varepsilon_{t}^{B}$ and $1-\varepsilon_{t}^{G^{-}}$, respectively (lines 12-14 and 16-18).

According to the specific characteristics of bags and graphs, we employ two different weighting strategies. For bags, they are the target of the classification objective and their genuine labels are given. Therefore, if a bag is misclassified by the current subgraph $g_{t}$ classifier $\mathcal{H}_{t}^{B}$, the bag weight is increased by using the weight coefficient factor $\beta_{t}^{B}$ (line 19) in order to find more informative subgraph in the next iteration to deal with those incorrectly predicted bags (line 21). This bag-level weighting mechanism is similar to the AdaBoost algorithm [25]. At individual graph level, because we propagate bag labels to graphs at the very beginning of the algorithm, some graphs in positive bags have been assigned with wrong labels. Therefore, if a graph in positive bags is misclassified (i.e. $\left.\mathcal{H}_{t}^{G}\left(G_{j}\right) \neq c\left(G_{j}\right)\right)$, in the next iteration we decrease its weight to reduce its effect through multiplying its weight by $\left(\beta^{G^{+}}\right)^{I\left(\mathcal{H}_{t}^{G}\left(G_{j}\right) \neq c\left(G_{j}\right)\right)} \in(0,1]$, where $\beta^{G^{+}}$is the underlying weight coefficient factor for positive graph (line 20). Thus in the next round the misclassified graphs in positive bags will bring less impact on the learning process than the current iteration (line 22). The graphs with large training weights will help the learning algorithm find better subgraphs. When tackling graphs in negative bags, the weight updating mechanism is the same with bags (line 23). This graph-level weighting mechanism is similar to the TrAdaBoost algorithm [48].

In the test phase, the test bag $B_{k}$ will be tested using a weighted classifier $\operatorname{sign}\left(\sum_{t=1}^{m} \beta_{t}^{B} \mathcal{H}_{t}^{B}\left(B_{k}\right)\right)$ by boosting all the $m$ weak classifiers $\mathcal{H}_{t}^{B}, t=1,2, \cdots, m$ to obtain its class label $y_{k}$ (line 25).

The key technical advantage of the bMGC process can be summarized as follows:

- Bag constrained subgraph mining: the two-level weight updating mechanism seamlessly integrates the unique bag- and graph-level constraints into a repetitive and progressive mining process. It helps explore informative subgraphs to represent multi-graph bags.

- Implicit feature representation: bMGC selects a subgraph to directly form a weak classifier in each iteration. This can efficiently tackle the challenge that no feature vectors are available for MGC.

- Generic boosting framework for multi-graph classification: the proposed framework solves multi-graph classification by exploring informative subgraphs as weak classifiers to form a strong boosting model. The framework can be easily adjusted to accommodate other types of graph or bag classifiers for multi-graph classification.

\section{EXPERIMENTS}

\section{A. DataSets}

1) DBLP Multi-graph Dataset: The DBLP dataset consists of bibliography data in computer science. We download a DBLP version called "DBLP-Citation-network V5" from Arnetminer (http://arnetminer.org/citation). Each record in DBLP is associated with a number of attributes including title, abstract, author names, year, venue, and reference names etc. [49]. To build multi-graph bags, we select papers published in Artificial Intelligence (AI), Computer Vision (CV), and Database (DB) fields to form multi-graph classification tasks. The goal is to predict which field a paper belongs to $\mathrm{AI}$ or $\mathrm{CV}$, $\mathrm{AI}$ or $\mathrm{DB}$, by using abstracts of each paper and the abstracts of its references. For each abstract, a fuzzy cognitive map (E-FCM) [50] based approach is used to extract a number of keywords and correlations between keywords. In our experiments, we use keywords as nodes and correlations between two keywords as edge weight values to build a graph. A threshold (0.005) is 
TABLE I

DBLP DATASET USED IN EXPERIMENTS

\begin{tabular}{cccc}
\hline Categories & Selected Conferences & \# of Papers & \# of Abstracts \\
\hline AI & $\begin{array}{c}\text { IJCAI,AAAI,NIPS,UAI,COLT, } \\
\text { ACL,KR,ICML,ECML,IJCNN }\end{array}$ & 800 & 4486 \\
\hline CV & $\begin{array}{c}\text { ICCV, CVPR, ECCV, ICPR, ICIP } \\
\text { ACM Multimedia, ICME }\end{array}$ & 800 & 4206 \\
\hline DB & $\begin{array}{c}\text { SIGMOD, PODS, VLDB, ICDE, CIKM } \\
\text { DASFAA, ICDT, SSD, DASFAA, }\end{array}$ & 800 & 4627 \\
\hline
\end{tabular}

used to remove edges whose correlation values are less than the threshold. At the last step, the graph is converted into an unweighted graph by setting the weight values of all remaining edges as " 1 ". The similar graph representation was also used in previous works [51], [52], [53], [54].

A conceptual view of building a multi-graph bag is shown in Figure 1. Notice that $\mathrm{AI}$ and $\mathrm{CV}, \mathrm{AI}$ and $\mathrm{DB}$ are overlapped in many aspects, such as machine learning, optimization and data mining, which make them challenging MGC tasks. The original DBLP dataset contains a significant number of papers without references. We choose 2400 papers, each of which containing 1 to 10 references, to form two MGC tasks: DBLP(AI vs. CV) with positive (AI) and negative (CV) bags, and DBLP(AI vs. DB) with positive (AI) and negative (DB) bags. The last two columns in Table I report the number of bags (papers) and graphs (abstracts) in each category.

2) NCI Chemical Compound Multi-graph Dataset: The NCI cancer screening database is commonly used as the benchmark for graph classification. We download two NCI datasets with ID 1 and 109 from PubChem (http://pubchem.ncbi.nlm.nih.gov). Each NCI dataset belongs to a bioassay task for anticancer activity prediction, where each chemical compound is represented as a graph, with atoms representing nodes and bonds denoting edges. A chemical compound is positive if it is active against the corresponding cancer, or negative otherwise. The original NCI datasets are highly imbalanced, with about $5 \%$ positive graphs, which is reasonable to generate our multi-graph bags. To build multigraph bags, we randomly select 1 to 4 positive graphs and several negative graphs to form a positive bag, and randomly select a number of negative graphs to form a negative bag. To reflect different specific circumstances of real-world problems, we design two NCI multi-graph datasets. One is $\mathrm{NCI}(1)$, which is generated from NCI dataset with ID 1 , and the other is NCI(109), which is generated from NCI dataset with ID 109. The number of graphs in each bag may vary from 1-10.

Table II summarizes the NCI(1) and NCI(109) datasets used in our experiments, where columns 4-5 show the numbers of positive and negative graphs in all multi-graph bags. In the NCI multi-graph classification, a bag of graphs can be regarded as a molecular group. Investigating the activity of a molecular group is meaningful in the bio-pharmaceutical field. Because labeling individual compounds is expensive and timeconsuming, it is desirable to design effective methods (bMGC) to label the bag (molecular group).
TABLE II

NCI CANCER SCREEN DATASETS: NCI(1) AND NCI(109).

\begin{tabular}{ccccc}
\hline Dataset & ID & \# of Bag & \# of Positive Graphs & \# of Negative Graphs \\
\hline NCI(1) & 1 & 800 & 1593 & 6614 \\
\hline NCI(109) & 109 & 800 & 1600 & 6998 \\
\hline
\end{tabular}

\section{B. Baseline Methods}

To demonstrate the effectiveness of our multi-graph classification framework, we compare the proposed bMGC with both supervised and unsupervised bag constrained subgraph selection methods in the traditional multi-instance (MI) learning framework, which are summarized as follows:

- Information gain based approach (IG+MI): In these methods, a set of frequent subgraph from graphs in all bags are first mined by gSpan [34]. A supervised feature selection based on Information Gain (IG) is used to select $m$ subgraphs with the highest IG scores. After obtaining the $m$ subgraphs, IG based multi-instance approach (IG+MI) utilizes the selected subgraphs to represent graphs in bags, so a bag of graphs are converted into a bag of instances, through which the existing multi-instance learning methods can be applied for MGC learning.

- Top- $k$ based approach (Topk+MI): We also compare with the unsupervised feature selection methods, which use frequency as evaluation criterion for subgraph selection based on gSpan [34]. The Top- $k$ subgraphs with the highest frequency from graphs in bags are selected. Top$k$ based multi-instance approach (Topk+MI) transforms each bag of graphs into a bag of instances for learning.

To compare our multi-graph classification framework bMGC's performance with multi-instance learning, two types of benchmark multi-instance classifiers, including boosting based (MIBoost and MIOptimallBall) and four different kinds of general approaches (CitationKNN, MIRI, MIEMDD, and MISMO), are used in our experiment. In the following, CitationKNN denotes a lazy learning based method, MIRI is an improvement of tree learning based approach, MIEMDD is an improved diverse density [26] based way, and MISMO is an implementation of support vector machine for MIL. The baseline MIL method used in our experiments and their abbreviations are listed as follows.

1) Boosting for MI Learning Approaches:

- MIBoost is an algorithm [24] inspired by AdaBoost that builds a series of weak classifiers (Decision Stump is used in our experiment) using a single instance learner based on appropriately reweighted versions of the input data.

- MIOptimalBall treats the weak hypotheses for AdaBoost as balls [28] and the classification is based on the distance to a reference point. More specifically, this method attempts to find a ball in instance space so that all instances of all negative bags are outside the ball and at least one instance of each positive bag is inside the ball.

2) General MI Learning Approaches:

- CitationKNN, a nearest-neighbor-based approach, measures the distance between bags using Hausdorff dis- 
TABLE III

PAIRWISE $t$-TeST ReSUlt OF bMGC $v s$. BOOSTING BASED MI LEARNing METHOdS ON DBLP (a) AND NCI (b) DATASETS. A, B, AND C DENOTE bMGC, IG+MI, AND TOPK+MI, ReSPeCtively. $\mathcal{H}_{1}$ AND $\mathcal{H}_{2}$ DENOTE MIBOost AND MiOptimalBall, Respectively.

(a) $t$-test on DBLP dataset

\begin{tabular}{cccc||cccc}
\hline \multicolumn{4}{c}{ DBLP(AI vs CV) } & \multicolumn{4}{c}{ DBLP(AI vs DB) } \\
\hline $\mathcal{H}$ & A-B & A-C & B-C & $\mathcal{H}$ & A-B & A-C & B-C \\
$\mathcal{H}_{1}$ & $1.65 \mathrm{E}-04$ & $7.83 \mathrm{E}-05$ & $\mathbf{4 . 3 5 E}-01$ & $\mathcal{H}_{1}$ & $9.97 \mathrm{E}-05$ & $6.54 \mathrm{E}-04$ & $6.53 \mathrm{E}-06$ \\
$\mathcal{H}_{2}$ & $1.73 \mathrm{E}-08$ & $1.74 \mathrm{E}-12$ & $5.21 \mathrm{E}-13$ & $\mathcal{H}_{2}$ & $2.15 \mathrm{E}-11$ & $2.39 \mathrm{E}-09$ & $\mathbf{6 . 0 2 E}-01$ \\
\hline
\end{tabular}
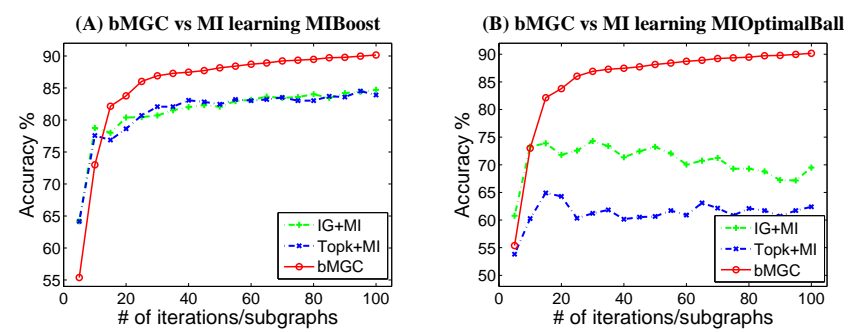

Fig. 4. Accuracy on DBLP(AI vs. CV) by using proposed bMGC and boosting based MI learning methods: (A) MIBoost and (B) MIOptimalBall.
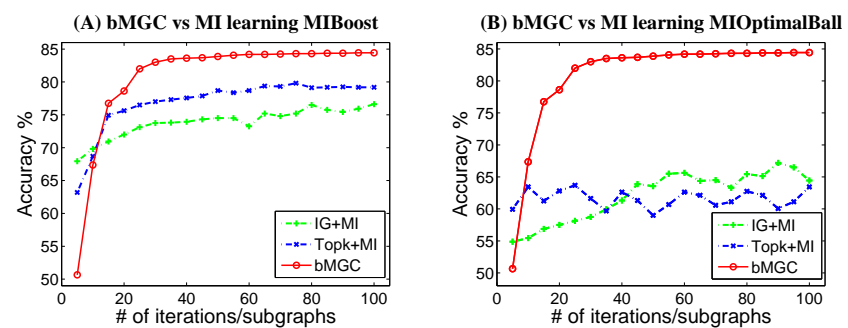

Fig. 5. Accuracy on DBLP(AI vs. DB) by using proposed bMGC and boosting based MI learning methods: (A) MIBoost and (B) MIOptimalBall.

tance [16]. The nearest neighbor example to be classified is the one nearest to both references and citers.

- MIEMDD is the expectation-maximization version of diverse density (DD) with the most-likely-cause model [26], which is used to find the most likely target points based on the DD model that has been learned [27].

- MIRI is a multi-instance classifier that utilizes partial MITI trees [17] with a single positive leaf to learn and represent rules. MIRI [18] is a simple modification to MITI to yield a rule learner for MIL.

- MISMO constructs a support vector machine classifier for multi-instance data [21], where the standard sequential minimization algorithm is used for support vector learning in conjunction with an MI kernel as described in [55].

\section{Experimental Settings}

In our experiments, all reported results are based on 10 times 10-fold cross-validation with classification accuracy being used as the performance metric. Unless specified otherwise, the default parameter settings are as follows: minimum support threshold min_sup $=4 \%$ for DBLP datasets and min_sup $=15 \%$ for NCI datasets. All the above classifiers for traditional multi-instance learning utilize the versions provided (b) $t$-test on NCI dataset

\begin{tabular}{cccc||cccc}
\hline \multicolumn{4}{c}{$\mathrm{NCI}(1)$} \\
\hline $\mathcal{H}$ & A-B & A-C & B-C & $\mathcal{H}$ & A-B & A-C & B-C \\
$\mathcal{H}_{1}$ & $1.87 \mathrm{E}-03$ & $1.03 \mathrm{E}-07$ & $3.85 \mathrm{E}-10$ & $\mathcal{H}_{1}$ & $5.79 \mathrm{E}-03$ & $8.21 \mathrm{E}-09$ & $1.53 \mathrm{E}-08$ \\
$\mathcal{H}_{2}$ & $1.84 \mathrm{E}-12$ & $8.63 \mathrm{E}-12$ & $\mathbf{2 . 0 8 E}-01$ & $\mathcal{H}_{2}$ & $9.38 \mathrm{E}-13$ & $1.94 \mathrm{E}-11$ & $\mathbf{6 . 8 0 E}-\mathbf{0 2}$ \\
\hline
\end{tabular}
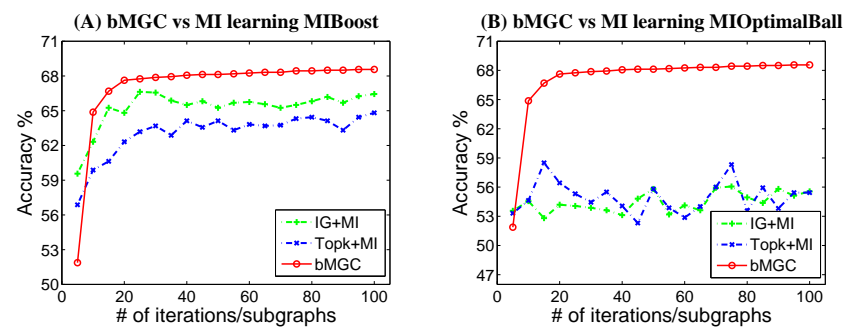

Fig. 6. Accuracy on NCI(1) by using proposed bMGC and boosting based MI learning methods: (A) MIBoost and (B) MIOptimalBall.
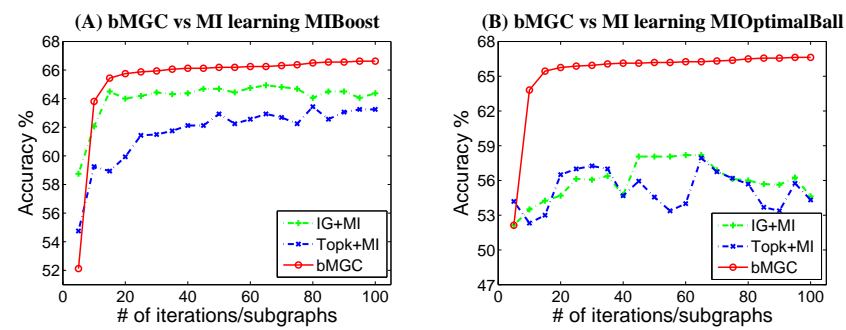

Fig. 7. Accuracy on NCI(109) by using proposed bMGC and boosting based MI learning methods: (A) MIBoost and (B) MIOptimalBall.

in WEKA machine learning workbench [56], with default parameter settings. Besides, all experiments are conducted on a Linux cluster computing node with an $\operatorname{Interl}(\mathrm{R}) \operatorname{Xeon}(\mathrm{R})$ @ 3.33GHZ CPU and 3GB memory.

\section{Accuracy on Multi-Graph Classification}

In this section, we report experimental results on DBLP and NCI datasets, by comparing the performance of bMGC with two types of multi-instance learning methods, including boosting based and general approaches under the supervised and unsupervised feature selection methods, respectively. We compare all methods in the setting that they have the same number of subgraphs. For our boosting based bMGC, one subgraph is selected in each iteration until the total number reaches $m$, whereas for those baseline methods, a number of $m$ subgraphs are selected in one time. As expected, bMGC clearly outperforms existing traditional MIL methods on both DBLP and NCI multi-graph datasets with different number of subgraphs (varying from 1 to 100).

1) bMGC vs. Boosting for MI Learning Approaches: We compare bMGC to MIBoost and MIOptimalBall, where the two boosting based baselines are two variants of the well known AdaBoost algorithm [25] with the objective of 

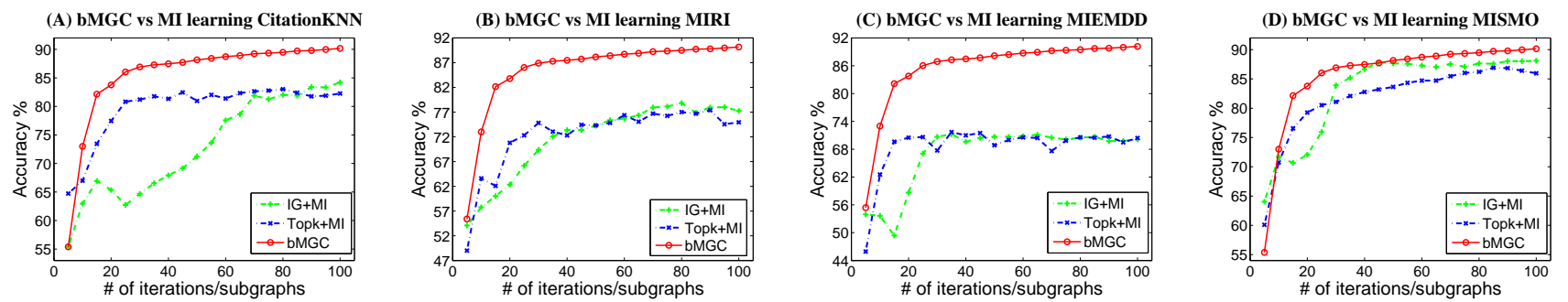

Fig. 8. Accuracy on DBLP(AI vs. CV) by using bMGC and generic MI learning methods: (A) CitationKNN; (B) MIRI; (C) MIEMDD, and (D) MISMO.
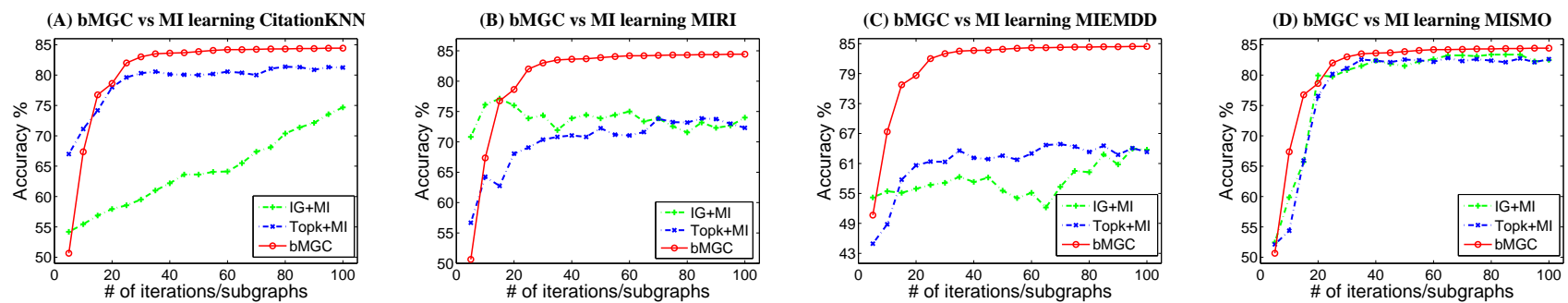

Fig. 9. Accuracy on DBLP(AI vs. DB) by using bMGC and generic MI learning methods: (A) CitationKNN; (B) MIRI; (C) MIEMDD, and (D) MISMO.
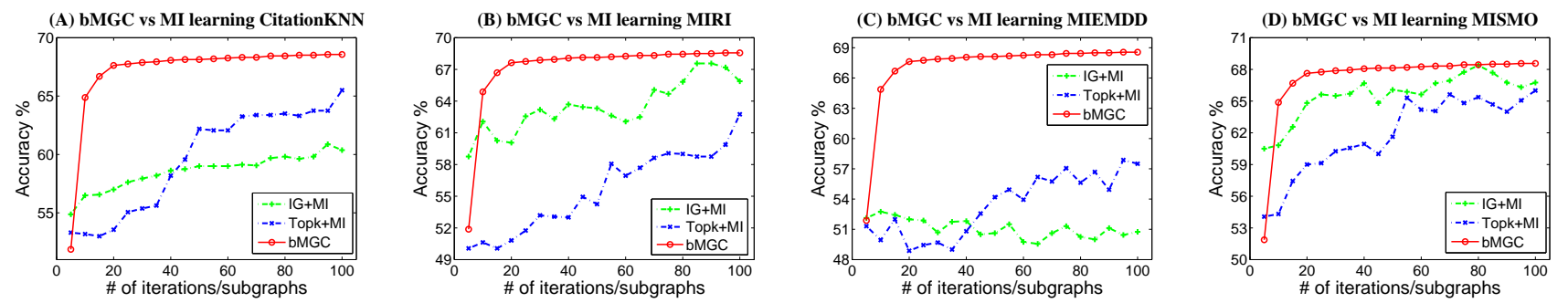

Fig. 10. Accuracy on NCI(1) by using bMGC and generic MI learning methods: (A) CitationKNN; (B) MIRI; (C) MIEMDD, and (D) MISMO.
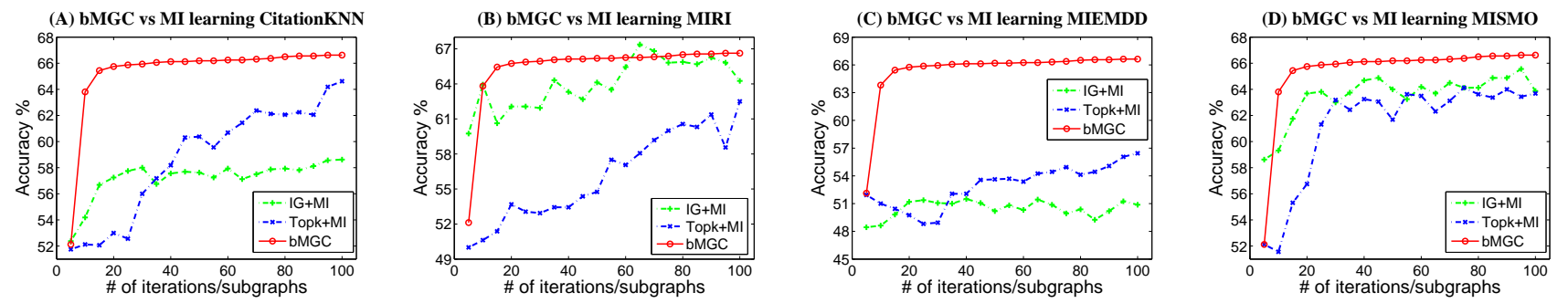

Fig. 11. Accuracy on NCI(109) by using bMGC and generic MI learning methods: (A) CitationKNN; (B) MIRI; (C) MIEMDD, and (D) MISMO.

TABLE IV

PAirwise $t$-TEST RESUlt OF bMGC $v s$. GENERAL MI LEARNing METHOdS ON DBLP (a) AND NCI (b) DATASETS. A, B, AND C DENOTE bMGC, IG+MI, AND TOPK+MI, ReSPeCtively. $\mathcal{H}_{1}, \mathcal{H}_{2}, \mathcal{H}_{3}$ and $\mathcal{H}_{4}$ DENOTE CitATIONKNN, MiRI, MiEMdD and MiSMO, RESPECTIVELY.

(a) $t$-test on DBLP dataset

\begin{tabular}{cccc||cccc}
\hline \multicolumn{4}{c}{ DBLP(AI vs CV) } & \multicolumn{4}{c}{ DBLP(AI vs DB) } \\
\hline $\mathcal{H}$ & A-B & A-C & B-C & $\mathcal{H}$ & A-B & A-C & B-C \\
$\mathcal{H}_{1}$ & $5.73 \mathrm{E}-08$ & $9.41 \mathrm{E}-07$ & $2.10 \mathrm{E}-04$ & $\mathcal{H}_{1}$ & $4.71 \mathrm{E}-10$ & $6.76 \mathrm{E}-03$ & $3.31 \mathrm{E}-11$ \\
$\mathcal{H}_{2}$ & $1.97 \mathrm{E}-11$ & $2.53 \mathrm{E}-15$ & $\mathbf{4 . 7 6 E - 0 1}$ & $\mathcal{H}_{2}$ & $1.12 \mathrm{E}-03$ & $2.24 \mathrm{E}-09$ & $3.63 \mathrm{E}-03$ \\
$\mathcal{H}_{3}$ & $3.95 \mathrm{E}-12$ & $3.04 \mathrm{E}-15$ & $\mathbf{2 . 6 6 E}-01$ & $\mathcal{H}_{3}$ & $2.97 \mathrm{E}-11$ & $4.60 \mathrm{E}-16$ & $5.08 \mathrm{E}-03$ \\
$\mathcal{H}_{4}$ & $2.01 \mathrm{E}-02$ & $3.99 \mathrm{E}-07$ & $\mathbf{1 . 1 8 E - 0 1}$ & $\mathcal{H}_{4}$ & $3.64 \mathrm{E}-03$ & $2.38 \mathrm{E}-03$ & $\mathbf{1 . 2 5 E}-01$ \\
\hline
\end{tabular}

minimizing the exponential loss for bags of instances. Like other boosting schemes, these two algorithms greedily fit an additive model to the training data. In each iteration of the sequential boosting process, a "weak" learner (Decision Stump (b) $t$-test on NCI dataset

\begin{tabular}{cccc||cccc}
\hline \multicolumn{4}{c}{$\mathrm{NCI}(1)$} & $\mathcal{H}$ & A-B & A-C & B-C \\
\hline $\mathcal{H}$ & A-B & A-C & B-C & $\mathcal{H}_{1}$ & NCI $(109)$ \\
$\mathcal{H}_{1}$ & $2.96 \mathrm{E}-11$ & $1.59 \mathrm{E}-07$ & $\mathbf{1 . 4 4 E - 0 1}$ & $\mathcal{H}_{1}$ & $2.11 \mathrm{E}-13$ & $2.95 \mathrm{E}-07$ & $\mathbf{7 . 0 3 E}-\mathbf{0 2}$ \\
$\mathcal{H}_{2}$ & $4.68 \mathrm{E}-05$ & $3.45 \mathrm{E}-11$ & $4.99 \mathrm{E}-12$ & $\mathcal{H}_{2}$ & $3.52 \mathrm{E}-02$ & $3.90 \mathrm{E}-10$ & $3.10 \mathrm{E}-11$ \\
$\mathcal{H}_{3}$ & $4.42 \mathrm{E}-13$ & $5.08 \mathrm{E}-12$ & $1.19 \mathrm{E}-02$ & $\mathcal{H}_{3}$ & $8.64 \mathrm{E}-16$ & $1.66 \mathrm{E}-12$ & $2.06 \mathrm{E}-04$ \\
$\mathcal{H}_{4}$ & $1.49 \mathrm{E}-02$ & $2.79 \mathrm{E}-07$ & $1.03 \mathrm{E}-07$ & $\mathcal{H}_{4}$ & $9.54 \mathrm{E}-04$ & $5.21 \mathrm{E}-06$ & $7.87 \mathrm{E}-04$ \\
\hline
\end{tabular}

for MIBoost, Ball for MIOptimalBall) is applied to generate one component of the underlying additive model.

Results in Figures 4(A) to 7(A) show that both bMGC and MIBoost can achieve a high accuracy on DBLP (AI vs. CV, 
AI vs. DB) and NCI $(1,109)$ datasets. Meanwhile, bMGC consistently outperforms MIBoost when the number of selected subgraphs is 20 or more. On the other hand, comparing our bMGC with MIOptimallBall, significant performance gain can be observed in Figures 4(B) to 7(B) on both datasets. The superior performance of $\mathrm{bMGC}$ is due to the optimal subgraph mining strategy combined with AdaBoost and TrAdaBoost algorithms. Further more, it seems that MIOptimallBall fails to adapt to the feature space composed of subgraphs.

Our results also show that bMGC has a very low accuracy in early iterations, and its accuracy may be worse than baselines such as MIBoost in some cases. This is mainly because that the boosting model of bMGC relies on weak classifiers to achieve better performance. As a result, when the number of weak classifiers is small (normally happens at the early stage of the boosting process), the accuracy of bMGC is noticeably low. In order to show that this situation will not affect the performance of bMGC, we summarize the pairwise $t$-test results (with confidence level $\alpha=0.05$ ) of bMGC and boosting MI learning methods on both datasets in Table III. Each entry (value) denotes the $p$-value for a $t$-test between two algorithms, and a $p$-value less than $\alpha=0.05$ indicates that the difference is statistically significant. From Table III, bMGC statistically outperforms boosting based MI learning baselines in all cases.

2) bMGC vs. General MI Learning Approaches: We carry out another experimental comparison to demonstrate the performance of bMGC, with other four different types of general MI learning approaches (CitationKNN, MIRI, MIEMDD and MISMO). From the results in Figures 8(C) to 11(C), MIEMDD shows ineffective performance for multi-graph classification, and increasing number of subgraphs may not obtain any additional accuracy gain.

Although the performance of CitationKNN, MIRI, and MISMO based methods improve as the number of subgraphs increases, they still cannot reach the best performance achieved by bMGC except for IG+MIRI on NCI(109) dataset as shown in Figure 11(B). It is also worth mentioning that bMGC may achieve comparable performances over other baselines in some cases, such as Topk+CitationKNN (Figure 9(A)) and MISMO (Figures 8(D) and 9(D)) on DBLP dataset, IG+MISMO (Figures 10(D) and 11(D)) on NCI dataset.

To furthermore validate the statistical performance of bMGC, in Table IV, we also report the pairwise $t$-test to validate the statistical significance between two methods. From Table IV, bMGC statistically outperforms general MI learning baselines in all cases. This is mainly attributed to the effectiveness of the proposed bag constrained subgraph exploration criterion and the specially designed boosting strategy, which weights a set of single weak classifiers under our specially designed weighting mechanism.

\section{E. Effectiveness of Subgraph Candidate Generation in bMGC}

As discussed above, one main component of bMGC is the utilization of generating subgraph candidates (as described in Section IV). More specifically, in addition to aggregating graphs in all bags $\mathcal{G}$, we also aggregate (1) graphs in all
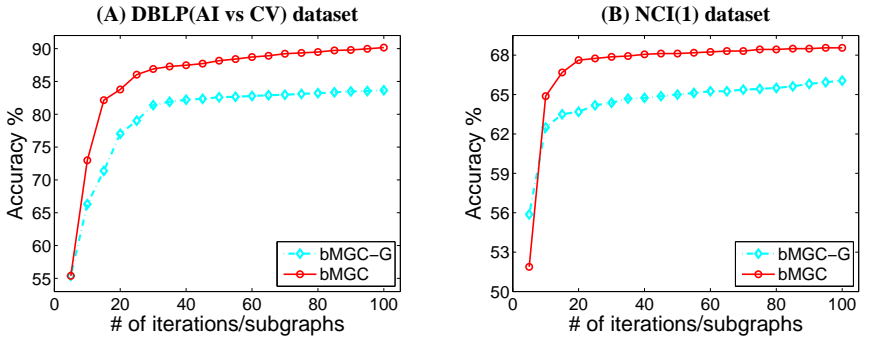

Fig. 12. Accuracy comparisons by using bMGC and bMGC-G on DBLP and NCI datasets, respectively.

positive bags $\mathcal{G}^{+}$, and (2) graphs in all negative bags $\mathcal{G}^{-}$. As a result, a set of diverse subgraph candidate patterns can be discovered for validation. In order to further illustrate the effectiveness of the proposed strategy for subgraph candidate generation and validate whether using the two extra graph sets $\mathcal{G}^{+}$and $\mathcal{G}^{-}$can indeed improve the performance of bMGC, we compare bMGC with an approach which only uses the $\mathcal{G}$ to generate the subgraphs for learning, namely bMGCG. In Figures 12(A) and 12(B), we report the accuracy with respect to different iterations on DBLP(AI $v s . \mathrm{CV})$ and $\mathrm{NCI}(1)$ datasets, respectively. The results show that the classification accuracy of bMGC using all three graph sets is normally $3 \%$ $5 \%$ higher than bMGC-G which only uses the $\mathcal{G}$. This is due to the fact that the separation of graphs into $\mathcal{G}^{+}$and $\mathcal{G}^{-}$can help find some unique subgraph patterns, which do not appear in the whole graph set $\mathcal{G}$. Indeed, because the subgraph exploration essentially relies on a threshold (i.e. the support value) to discover frequent subgraphs. When aggregating all graphs in one set $\mathcal{G}$, it is possible that a good subgraph in $\mathcal{G}^{+}$may not be discovered from $\mathcal{G}$, simply because the frequency of the subgraph is below the given threshold in $\mathcal{G}$. The separation of graphs into three sets $\mathcal{G}^{+}, \mathcal{G}^{-}$, and $\mathcal{G}$ will therefore help discover a rich set of subgraph candidates, through which bMGC can fine the ones with the highest informative scores.

\section{F. Convergence Study}

Figure 13 reports the error rate curves of bMGC in terms of the number of iterations on four multi-graph datasets. The curves are quite smooth, but converge well, which is consistent with the theoretical analysis and the existing observations from Adaboost [25]. The error rates of bMGC, after the algorithm reaches the convergence, are higher on DBLP datasets than on the NCI datasets. Overall, bMGC on all four datasets receives a fast convergence speed.

For NCI datasets, the convergence is reached within 10 iterations, whereas for DBLP datasets, bMGC's convergence is reached after 20 or more iterations. Notice that each weak classifier in bMGC denotes one subgraph, this indicates that more subgraph features are needed in order to differentiate the object classes in the DBLP dataset. Indeed, because DBLP tasks involve some overlapping domains (such as AI vs. CV), using more subgraph features (which correspond to keywords and their correlations) can constantly help improve the classification accuracy. For NCI graphs, the positive vs. negative graphs are mostly separated by some unique subgraph 


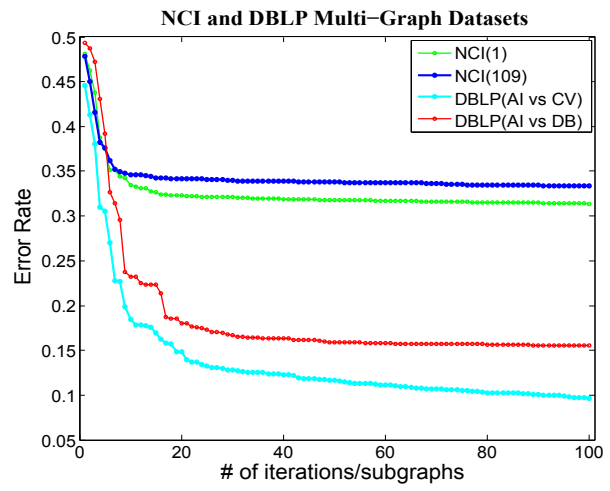

Fig. 13. The error rate curves on DBLP (AI vs. CV, AI vs. DB) and NCI(1 and 109) multi-graph datasets in terms of the number of iterations.

features. So as long as such unique patterns are discovered, the algorithm can quickly converge.

\section{G. Effectiveness Results}

To evaluate the effectiveness of the pruning module of bMGC in reducing the search space (as described in Section $\mathrm{V}-\mathrm{C}$ ), we compare bMGC with an approach which does not have pruning module in the subgraph search space (denoted by ubMGC). In our implementation, ubMGC first uses gSpan to find a set of frequent subgraphs, and then selects the optimal subgraph by using the same criteria as bMGC in each iteration. In Figures 14(A) and 14(B), we report the average CPU runtime performance with respect to different minimum support values min_sup (the number of selected subgraphs is fixed to 100) on $\operatorname{DBLP}(\mathrm{AI} v s$. CV) and $\mathrm{NCI}(1)$ datasets, respectively. The results show that as the min_sup values increase, the runtime of both pruning and unpruning bMGC decrease, this is mainly because a larger min_sup value will reduce the number of candidates for validation. Accordingly, by incorporating the proposed pruning strategy, bMGC can improve the runtime performance. The reason is that the bScore upper bound of bMGC can effectively help prune the subgraph search space without decreasing the quality of classification.

\section{DISCUSSION}

In this paper, we focus on using subgraph based boosting framework for multi-graph classification. Indeed, the idea
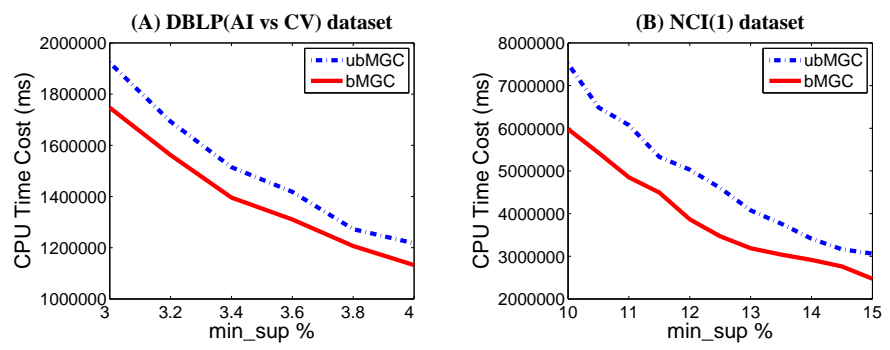

Fig. 14. Average CPU runtime for bMGC $v s$ unpruned ubMGC with different min_sup under a fixed number of subgraphs $m=100$ on DBLP and NCI datasets, respectively. of exploiting subgraphs for graph classification has been studied in a number of existing works, including a recent ensemble based semi-supervised graph stream classification approach [9]. The core of the proposed bMGC approach is to combine two types of boosting strategies: AdaBoost [25] for bag-level boosting and TrAdaBoost [48] for graphlevel boosting, to integrate graph- and bag-level learning for multi-graph classification. Boosting algorithms for graph classification have already been studied in number of previous works. For example, Kudo et al. [41] proposes an AdaBoost based graph classification approach, which is the original and the best known algorithm among many variants [42], [43], [44]. Meanwhile, LPBoost [57], namely linear programming Boosting, is another type of boosting algorithm for graph classification. The proposed bMGC follows similar subgraph search approaches as used in these existing works. For bMGC, it uses gSpan algorithm [34] in each boosting iteration, together with the proposed pruning strategy, to explore subgraphs.

The main complication of multi-graph classification is that the genuine labels of graphs inside a positive bag are unknown. To tackle uncertainty inside positive bags, bMGC takes the bag constraints into consideration and explores subgraphs to represent graphs with maximum diversity, as defined in Eq. (2). This is similar to the way of handling unlabeled graphs in an existing semi-supervised graph stream classification method [9]. In [9], an instance weighting mechanism has also been proposed but is different from the weighting approach in bMGC, where the weights are directly associated to the graphs and bags. In addition, the weight updating strategy in [9] is based on AdaBoost [25], which only considers labeled graphs. In bMGC, we borrow the weighting strategy from TrAdaBoost [48] to update the graph weighs in both labeled and unlabeled graph sets. In summary, the idea in [9] provides inspirations to motivate the proposed multi-graph classification design.

We believe that the proposed bMGC opens a new opportunity to expand existing multi-instance learning to increasingly popular graph applications. Although bMGC proposes to use subgraph mining to tackle the MGC challenges, the principle of combining graph and bag level constraints can be extended to many other types of approaches to handle MGC problems. For example, for kernel based methods, MGC problem can be solved by two subtasks: (1) add multi-graph constraints to traditional graph kernel, and (2) propose a new multigraph kernel framework. In addition, one can also impose multi-graph constrains to graph embedding methods (e.g. the one in [32]) to directly calculate the distance between two graphs or between two graph bags. With the calculated distances between graphs and between bags, standard learning algorithms (including MIL algorithms) can be applied to solve multi-graph classification tasks.

\section{CONCLUSION}

In this paper, we investigated a novel Multi-Graph Classification (MGC) problem, in which a number of graphs form a bag, with each bag being labeled as either positive or negative. Multi-graph representation can be used to represent many real-world applications, where label is only available 
for a bag of objects with complex structures. To build a learning model for multi-graph classification, we proposed a boosting based multi-graph classification framework (bMGC), which employs dynamic weight adjustment, at both graphand bag-levels, to select one subgraph in each iteration to form a set of weak graph classifiers. The multi-graph classification is achieved by using weighted combination of weak graph classifiers. Experiments on two real-world multi-graph classification tasks, including DBLP citation network and NCI chemical compound classification, demonstrate that our method is effective in finding informative subgraph, and its accuracy is significantly better than baseline methods.

\section{APPENDIX A}

\section{PROOF OF THE THEOREM 1}

According to Eq. (8), for any $g_{k}^{\prime} \supseteq g_{k}$ we have

$$
\begin{aligned}
r\left(g_{k}^{\prime}\right)= & \boldsymbol{f}_{g_{k}^{\prime}}^{\top} Q \boldsymbol{f}_{g_{k}^{\prime}} \\
= & {\left[\left(\boldsymbol{f}_{g_{k}^{\prime}}^{B}\right)^{\top}\left(\boldsymbol{f}_{g_{k}^{\prime}}^{G}\right)^{\top}\right]\left[\begin{array}{cc}
Q_{B} & 0 \\
0 & Q_{G}
\end{array}\right]\left[\begin{array}{c}
\boldsymbol{f}_{g_{k}^{\prime}}^{B} \\
\boldsymbol{f}_{g_{k}^{\prime}}^{G}
\end{array}\right] } \\
= & \left(\boldsymbol{f}_{g_{k}^{\prime}}^{B}\right)^{\top} Q_{B} \boldsymbol{f}_{g_{k}^{\prime}}^{B}+\left(\boldsymbol{f}_{g_{k}^{\prime}}^{G}\right)^{\top} Q_{G} \boldsymbol{f}_{g_{k}^{\prime}}^{G} \sum_{i, j: B_{i}, B_{j} \in \mathcal{B}\left(g_{k}^{\prime}\right)} Q_{i j}^{B}+\sum_{i, j: G_{i}, G_{j} \in \mathcal{G}\left(g_{k}^{\prime}\right)} Q_{i j}^{G}
\end{aligned}
$$

where $\mathcal{B}\left(g_{k}^{\prime}\right) \triangleq\left\{B_{i} \mid g_{k}^{\prime} \subseteq G_{j} \in B_{i}, 1 \leq i \leq p, 1 \leq j \leq\right.$ $q\}$ and $\mathcal{G}\left(g_{k}^{\prime}\right) \triangleq\left\{G_{j} \mid g_{k}^{\prime} \subseteq G_{j}, 1 \leq j \leq q\right\}$. Since $g_{k}^{\prime}$ is the supergraph of $g_{k}$ (i.e. $g_{k}^{\prime} \supseteq g_{k}$ ), according to the antimonotonic property, we have $\mathcal{B}\left(g_{k}^{\prime}\right) \subseteq \mathcal{B}\left(g_{k}\right)$ and $\mathcal{G}\left(g_{k}^{\prime}\right) \subseteq$ $\mathcal{G}\left(g_{k}\right)$.

$$
\begin{aligned}
r\left(g_{k}^{\prime}\right) & =\sum_{i, j: B_{i}, B_{j} \in \mathcal{B}\left(g_{k}^{\prime}\right)} Q_{i j}^{B}+\sum_{i, j: G_{i}, G_{j} \in \mathcal{G}\left(g_{k}^{\prime}\right)} \hat{Q}_{i j}^{G} \\
& \leq \sum_{i, j: B_{i}, B_{j} \in \mathcal{B}\left(g_{k}^{\prime}\right)} \hat{Q}_{i j}^{B}+\sum_{i, j: G_{i}, G_{j} \in \mathcal{G}\left(g_{k}^{\prime}\right)} \hat{Q}_{i j}^{G} \\
& \leq \sum_{i, j: B_{i}, B_{j} \in \mathcal{B}\left(g_{k}\right)} \hat{Q}_{i j}^{B}+\sum_{i, j: G_{i}, G_{j} \in \mathcal{G}\left(g_{k}\right)} \hat{Q}_{i j}^{G} \\
& =\left(\boldsymbol{f}_{g_{k}}^{B}\right)^{\top} \hat{Q}_{B} \boldsymbol{f}_{g_{k}}^{B}+\left(\boldsymbol{f}_{g_{k}}^{G}\right)^{\top} \hat{Q}_{G} \boldsymbol{f}_{g_{k}}^{G} \\
& =\boldsymbol{f}_{g_{k}}^{\top} \hat{Q} \boldsymbol{f}_{g_{k}}=\hat{r}\left(g_{k}\right)
\end{aligned}
$$

Thus, for any $g_{k}^{\prime} \supseteq g_{k}, r\left(g_{k}^{\prime}\right) \leq \hat{r}\left(g_{k}\right)$.

\section{REFERENCES}

[1] M. Deshpande, M. Kuramochi, N. Wale, and G. Karypis, "Frequent substructure-based approaches for classifying chemical compounds," IEEE Trans. on Knowl. and Data Eng., vol. 17, pp. 1036-1050, 2005.

[2] W. Lian, D.-L. Cheung, N. Mamoulis, and S.-M. Yiu, "An efficient and scalable algorithm for clustering xml documents by structure," IEEE Trans. on Knowl. and Data Eng., vol. 16, no. 1, pp. 82-96, 2004.

[3] C. Chen, C. X. Lin, M. Fredrikson, M. Christodorescu, X. Yan, and J. Han, "Mining graph patterns efficiently via randomized summaries," in Proc. 35th International Conference on Veay Large Data Bases (VLDB), 2009, pp. 742-753.

[4] H. Wang, H. Huang, and C. Ding, "Image categorization using directed graphs," in Proc. 11th European Conference on Computer Vision (ECCV), 2010, pp. 762-775.

[5] R. Angelova and G. Weikum, "Graph-based text classification: learn from your neighbors," in Proc. 29th annual international ACM SIGIR conference on Research and development in information retrieval (SIGIR), 2006, pp. 485-492.
[6] Z. Harchaoui and F. Bach, "Image classification with segmentation graph kernels," in Proc. 20th IEEE Conference on Computer Vision and Pattern Recognition (CVPR), 2007, pp. 1-8.

[7] M. Kuramochi and G. Karypis, "Frequent subgraph discovery," in Proc. 1st International Conference on Data Mining (ICDM), 2001, pp. 313320.

[8] M. Thoma, H. Cheng, A. Gretton, J. Han, H. Kriegel, A. Smola, L. Song, P. Yu, X. Yan, and K. Borgwardt, "Near-optimal supervised feature selection among frequent subgraphs," in Proc. 9th SIAM International Conference on Data Mining (SDM), 2009, pp. 1075-1086.

[9] S. Pan, X. Zhu, C. Zhang, and P. Yu, "Graph stream classification using labeled and unlabeled graphs," in Proc. 29th IEEE International Conference on Data Engineering (ICDE), 2013, pp. 398-409.

[10] R. Kohavi and G. H. John, "Wrappers for feature subset selection," Artif. Intell., vol. 97, no. 1-2, pp. 273-324, 1997.

[11] T. Dietterich., R. Lathrop, and T. Lozano-Pérez, "Solving the multiple instance problem with axis-parallel rectangles," Artif. Intell., vol. 89, pp. 31-71, 1997.

[12] Z. Fu, A. Robles-Kelly, and J. Zhou, "Milis: Multiple instance learning with instance selection," IEEE Trans Pattern Anal Mach Intell., vol. 33, no. 5, pp. 958-977, 2011

[13] Z.-H. Zhou, K. Jiang, and M. Li, "Multi-instance learning based web mining," Applied Intelligence, vol. 22, no. 2, pp. 135-147, 2005.

[14] D. Kelly, J. McDonald, and C. Markham, "Weakly supervised training of a sign language recognition system using multiple instance learning density matrices," Trans. Sys. Man Cyber. Part B, vol. 41, no. 2, pp. 526-541, 2011.

[15] Z. Zhou, M. Zhang, S. Huang, and Y. Li, "Multi-instance multi-label learning," Artificial Intelligence, vol. 176, pp. 2291-2320, 2012.

[16] J. Wang, "Solving the multiple-instance problem: A lazy learning approach," in Proc. 17th International Conference on Machine Learning (ICML), 2000, pp. 1119-1125.

[17] H. Blockeel and A. Srinivasan, "Multi-instance tree learning," in Proc. 22th International Conference on Machine Learning (ICML), 2005, pp. $57-64$.

[18] L. Bjerring and E. Frank, "Beyond trees: Adopting miti to learn rules and ensemble classifiers for multi-instance data," in Proc. 24th International Conference on Advances in Artificial Intelligence (AI), 2011, pp. 41-50.

[19] Y. Chevaleyre and J. Zucker, "A framework for learning rules from multiple instance data," in Proc. 12th European Conference on Machine Learning (ECML), 2001, pp. 49-60.

[20] M. Zhang and Z. Zhou, "Improve multi-instance neural networks through feature selection," Neural Process. Lett., vol. 19, pp. 1-10, 2004

[21] X. Qi and Y. Han, "Incorporating multiple SVMs for automatic image annotation," Pattern Recogn., vol. 40, pp. 728-741, 2007.

[22] S. Ray and M. Craven, "Supervised versus multiple instance learning: an empirical comparison," in Proc. the 22nd International Conference on Machine Learning (ICML), 2005, pp. 697-704.

[23] H. Yuan, M. Fang, and X. Zhu, "Hierarchical sampling for multi-instance ensemble learning," IEEE Trans. on Knowl. and Data Eng., vol. 25, no. 12 , pp. 2900-2905, 2013

[24] X. Xu and E. Frank, "Logistic regression and boosting for labeled bags of instances," in Proc. 8th Pacific-Asia Conference on Knowledge Discovery and Data Mining (PAKDD), 2004, pp. 272-281.

[25] M. Telgarsky, "A primal-dual convergence analysis of boosting," $J$. Mach. Learn. Res., vol. 13, pp. 561-606, 2012.

[26] O. Maron and T. Lozano-Pérez, "A framework for multiple-instance learning," in Proc. 12th Annual Conference on Neural Information Processing Systems (NIPS), 1998, pp. 570-576.

[27] Q. Zhang and S. Goldman, "Em-dd: An improved multiple-instance learning technique," in Proc. 15th Annual Conference on Neural Information Processing Systems (NIPS), 2001, pp. 1073-1080.

[28] P. Auer and R. Ortner, "A boosting approach to multiple instance learning," in Proc. 15th European Conference on Machine Learning (ECML), 2004, pp. 63-74.

[29] J. Wu, X. Zhu, C. Zhang, and Z. Cai, "Multi-instance multi-graph dual embedding learning," in Proc. 13th International Conference on Data Mining (ICDM), 2013, pp. 827-836.

[30] S. V. N. Vishwanathan, K. M. Borgwardt, R. I. Kondor, and N. N. Schraudolph, "Graph kernels," J. Mach. Learn. Res., vol. 11, pp. 12011242, 2008.

[31] P. Mahe, N. Ueda, T. Akutsu, J. Pettet, and J. Vert, "Extensions of marginalized graph kernels," in Proc. the 21st International Conference on Machine Learning (ICML), 2004, pp. 552-559.

[32] K. Riesen and H. Bunke, "Graph classification by means of lipschitz embedding," IEEE Trans. Sys. Man Cyber. Part B, vol. 39, pp. 1472 $1483,2009$. 
[33] — Graph Classification and Clustering Based on Vector Space Embedding. World Scientific Publishing Co., Inc., 2010.

[34] X. Yan and J. Han, "gspan: Graph-based substructure pattern mining," in Proc. 2nd International Conference on Data Mining (ICDM), 2002, pp. 721-724.

[35] A. Inokuchi, T. Washio, and H. Motoda, "An apriori-based algorithm for mining frequent substructures from graph data," in Proc. 4th European Conference on Principles and Practice of Knowledge Discovery in Databases (PKDD), 2000, pp. 13-23.

[36] C. Borgelt and M. Berthold, "Mining molecular fragments: Finding relevant substructures of molecules," in Proc. 2nd International Conference on Data Mining (ICDM), 2002, pp. 51-58.

[37] S. Nijssen and J. Kok, "A quickstart in frequent structure mining can make a difference," in Proc. 10th ACM SIGKDD International Conference on Knowledge Discovery and Data Mining (KDD), 2004, pp. 647-652.

[38] X. Yan, H. Cheng, J. Han, and P. S. Yu, "Mining significant graph patterns by leap search," in Proc. 27th ACM SIGMOD International Conference on Management of Data (SIGMOD), 2008, pp. 433-444.

[39] H. Saigo, N. Krämer, and K. Tsuda, "Partial least squares regression for graph mining," in Proc. 14th ACM SIGKDD International Conference on Knowledge Discovery and Data Mining (KDD), 2008, pp. 578-586.

[40] N. Jin, C. Young, and W. Wang, "Gaia: graph classification using evolutionary computation," in Proc. 29th ACM SIGMOD International Conference on Management of Data (SIGMOD), 2010, pp. 879-890.

[41] T. Kudo, E. Maeda, and Y. Matsumoto, "An application of boosting to graph classification," in Proc. 18th Annual Conference on Neural Information Processing Systems (NIPS), 2004, pp. 729-736.

[42] S. Nowozin, K. Tsuda, T. Uno, T. Kudo, and G. Bakir, "Weighted substructure mining for image analysis," in Proc. 20th IEEE Conference on Computer Vision and Pattern Recognition (CVPR), 2007, pp. 1-8.

[43] H. Saigo, S. Nowozin, T. Kadowaki, T. Kudo, and K. Tsuda, "gboost: A mathematical programming approach to graph classification and regression," Mach. Learn., vol. 75, no. 1, pp. 69-89, 2009.

[44] S. Pan and X. Zhu, "Graph classification with imbalanced class distributions and noise," in Proc. 23rd International Joint Conference on Artificial Intelligence (IJCAI), 2013, pp. 1586-1592.

[45] H. Fei and J. Huan, "Boosting with structure information in the functional space: An application to graph classification," in Proc. 16th ACM SIGKDD Conference on Knowledge Discovery and Data Mining (KDD), 2010, pp. 643-652.

[46] B. Zhang, G. Ye, Y. Wang, W. Wang, J. Xu, G. Herman, and Y. Yang, "Multi-class graph boosting with subgraph sharing for object recognition," in Proc. 20th International Conference on Pattern Recognition (ICPR), 2010, pp. 1541-1544.

[47] M. Grbovic, C. Dance, and S. Vucetic, "Sparse principal component analysis with constraints," in Proc. 26th Conference on Artificial Intelligence (AAAI), 2012, pp. 935-941.

[48] W. Dai, Q. Yang, G. Xue, and Y. Yu, "Boosting for transfer learning," in Proc. 24th International Conference on Machine Learning (ICML), 2007, pp. 193-200.

[49] J. Tang, J. Zhang, L. Yao, J. Li, L. Zhang, and Z. Su, "Arnetminer: extraction and mining of academic social networks," in Proc. 14th ACM SIGKDD International Conference on Knowledge Discovery and Data Mining (KDD), 2008, pp. 990-998.

[50] K. Perusich and M. McNeese, "Using fuzzy cognitive maps for knowledge management in a conflict environment," Trans. Sys. Man Cyber Part C, vol. 36, no. 6, pp. 810-821, 2006.

[51] X. L. Q. Hu, W. Xu, and Z. Yu, "Discovery of textual knowledge flow based on the management of knowledge maps," Concurr. Comput. : Pract. Exper., vol. 20, no. 15, pp. 1791-1806, 2008.

[52] X. Luo, Z.X., J. Yu, and X. Chen, "Building association link network for semantic link on web resources," IEEE Trans. on Autmom Sci. and Eng., vol. 8, no. 3, pp. 482-494, 2011.

[53] J. Wu, Z. Hong, S. Pan, X. Zhu, C. Zhang, and Z. Cai, "Multi-graph learning with positive and unlabeled bags," in Proc. the 14th SIAM International Conference on Data Mining (SDM), 2014, pp. 217-225.

[54] J. Wu, X. Zhu, C. Zhang, and P. Yu, "Bag constrained structure pattern mining for multi-graph classification,” Knowledge and Data Engineering, IEEE Transactions on, vol. PP, no. 99, pp. 1-1, 2014

[55] T. Gartner, P. A. Flach, A. Kowalczyk, and A. J. Smola, "Multi-instance kernels," in Proc. 19th International Conference on Machine Learning (ICML), 2002, pp. 179-186.

[56] I. H. Witten and E. Frank, Data Mining: Practical Machine Learning Tools and Techniques, Second Edition (Morgan Kaufmann Series in Data Management Systems). Morgan Kaufmann Publishers Inc., 2005.
[57] A. Demiriz, K. P. Bennett, and J. Shawe-Taylor, "Linear programming boosting via column generation," Mach. Learn., vol. 46, no. 1-3, pp. 225-254, 2002.

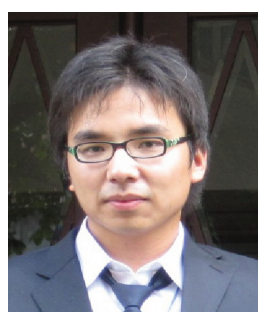

Jia Wu received his bachelor degree in computer science from China University of Geosciences (CUG), Wuhan, China, in 2009. Since September 2009 , he has been working toward the $\mathrm{PhD}$ degree under the Master-Doctor combined program in computer science from CUG. Besides, he is also pursuing a $\mathrm{PhD}$ degree in the Centre for Quantum Computation and Intelligent Systems, Faculty of Engineering and Information Technology, University of Technology, Sydney (UTS), Australia. His research focuses on data mining and machine learning.

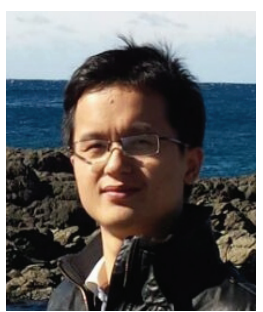

Shirui Pan received his master degree in computer science from Northwest A\&F University, Yangling, Shaanxi, China, in 2011. Since September 2011, he has been working toward the PhD degree in the Centre for Quantum Computation and Intelligent Systems, Faculty of Engineering and Information Technology, University of Technology, Sydney (UTS). His research focuses on data mining and machine learning.

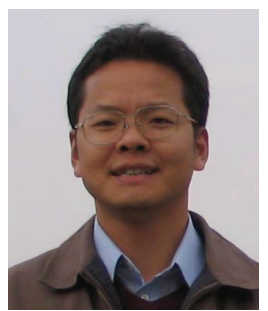

Xingquan Zhu received the $\mathrm{PhD}$ degree in computer science from Fudan University, Shanghai, China. $\mathrm{He}$ is an associate professor in the Department of Computer \& Electrical Engineering and Computer Science, Florida Atlantic University. Prior to that, he was with the Centre for Quantum Computation \& Intelligent Systems, University of Technology, Sydney, Australia. His research interests mainly include data mining, machine learning, and multimedia systems. Since 2000, he has published more than 170 refereed journal and conference papers in these areas, including two Best Paper Awards and one Best Student Paper Award. Dr. Zhu was an associate editor of the IEEE Transactions on Knowledge and Data Engineering (2008-2012), and is currently serving on the Editor Board of International Journal of Social Network Analysis and Mining SNAM (2010date) and Network Modeling Analysis in Health Informatics and Bioinformatics Journal (2014-date). He served or is serving as a program committee co-chair for the 14th IEEE International Conference on Bioinformatics and BioEngineering (BIBE-2014), IEEE International Conference on Granular Computing (GRC-2013), 23rd IEEE International Conference on Tools with Artificial Intelligence (ICTAI-2011), and the 9th International Conference on Machine Learning and Applications (ICMLA-2010). He also served as a conference co-chair for ICMLA-2012. He is a senior member of the IEEE.

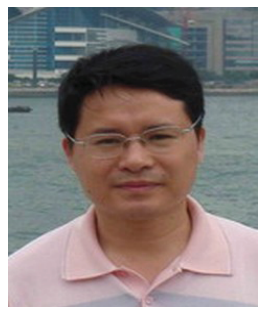

Zhihua Cai received the B.Sc. degree from Wuhan University, Wuhan, China, in 1986, the M.Sc. degree from Beijing University of Technology, Beijing, China, in 1992, and the Ph.D. degree from China University of Geosciences, Wuhan, in 2003. He is currently a faculty member with the School of Computer Science, China University of Geosciences. He has published over 50 research papers in journals and international conferences, such as IEEE Transactions on Knowledge and Data Engineering, IEEE Transactions on Cybernetics, Applied Soft Computing, Information Sciences, Knowledge-Based Systems, Knowledge and Information Systems, etc. His main research areas include data mining, machine learning, evolutionary computation, and their applications. 